\title{
The helium content and age of the Hyades:
}

\section{Constraints from five binary systems and Hipparcos parallaxes}

\author{
Y. Lebreton ${ }^{1}$, J. Fernandes ${ }^{2}$, and T. Lejeune ${ }^{2}$ \\ 1 DASGAL, CNRS UMR 8633, Observatoire de Paris, Place J. Janssen, 92195 Meudon, France \\ 2 Observatório Astronómico da Universidade de Coimbra, 3040 Coimbra, Portugal
}

Received 4 April 2001 / Accepted 17 May 2001

\begin{abstract}
We compare the accurate empirical mass-luminosity (M-L) relation based on five Hyades binary systems to predictions of stellar models calculated with various input parameters (helium, metallicity and age) or physics (mixing-length ratio, model atmosphere, equation of state and microscopic diffusion). Models based on a helium content $Y \sim 0.28$ inferred from the $\Delta Y / \Delta Z$ enrichment law are more than $3 \sigma$ beyond the observations, suggesting that the Hyades initial helium abundance is lower than expected from its supersolar metallicity. With the photometric metallicity $([\mathrm{Fe} / \mathrm{H}]=0.144 \pm 0.013 \mathrm{dex}$, Grenon 2000) we derive $Y=0.255 \pm 0.009$. Because of the $(Y,[\mathrm{Fe} / \mathrm{H}])$ degeneracy in the M-L plane, the uncertainty grows to $\Delta Y=0.013$ if the metallicity from spectroscopy is adopted $([\mathrm{Fe} / \mathrm{H}]=0.14 \pm 0.05 \mathrm{dex}$, Cayrel de Strobel et al. 1997). We use these results to discuss the Hertzsprung-Russell (HR) diagram of the Hyades, in the $\left(M_{V}, B-V\right)$ plane, based on the very precise Hipparcos dynamical parallaxes. Present models fit the tight observed sequence very well except at low temperatures. We show that the HR diagram does not bring further constraints on the helium abundance or metallicity of the cluster. In the low mass region of the HR diagram sensitive to the mixing-length parameter $\left(\alpha_{\mathrm{MLT}}\right)$, the slope of the main sequence (MS) suggests that $\alpha_{\text {MLT }}$ could decrease from a solar (or even supersolar) value at higher mass to subsolar values at low mass, which is also supported by the modeling of the vB22 M-L relation. We find that the discrepancy at low temperatures $((B-V) \gtrsim 1.2)$ remains, even if an improved equation of state or better model atmospheres are used. Finally, we discuss the positions of the stars at turn-off in the light of their observed rotation rates and we deduce that the maximum age of the Hyades predicted by the present models is $~ 650$ Myr.
\end{abstract}

Key words. open clusters and associations: Hyades - stars: fundamental parameters - stars: interiors stars: Hertzsprung-Russell (HR) and C-M diagram - stars: individual: vB22, $\theta^{2}$ Tau - stars: rotation

\section{Introduction}

Open clusters provide information and strong constraints for the stellar evolution theory. They give the opportunity to study large numbers of stars spanning a broad range of masses and evolutionary stages and that can be assumed to have similar age and chemical composition. Depending on the cluster studied, observations may give 1) the position of the cluster sequence in the HR diagram, 2) the density of stars along that sequence, 3) the M-L relation if the masses of some binary stars are accessible to observation. The analysis of the observational features of a given cluster by means of internal structure models allows estimating characteristics not directly accessible through observation, such as the age or helium content of the members. Furthermore, if the observational data are accurate enough, constraints on the physical processes at work in the stellar interiors, for instance the various transport processes, can be inferred.

Send offprint requests to: Y. Lebreton, e-mail: Yveline.Lebreton@obspm.fr
The Hyades is the nearest moderately rich star cluster. It has served for a long time to define absolute magnitude calibrations and, in turn, to fix the zero-point of the galactic and extragalactic distance scales. Also, as an open cluster, the precise knowledge of its chemical composition and age is fundamental for studies of the kinematic and chemical evolution of our Galaxy. The metallicity [Fe/H] (logarithm of the number abundances of iron to hydrogen relative to the solar value) is accessible through photometric or spectroscopic observations (see Sect. 2.1). On the other hand, the Hyades dwarfs are too cool for helium lines to be visible in their spectra and their helium content to be determined directly. The helium abundance and age have to be derived from the analysis of the observations using stellar models.

Recently, high-quality observations of the Hyades stars have been obtained. Hipparcos data combined with ground-based photometric or spectroscopic observations provided a more precise extended HR diagram of the cluster (Perryman et al. 1998; Dravins et al. 1997; de Bruijne et al. 2000). On the other hand, observations of several 
binary systems in the Hyades yielded a much improved M-L relation (Torres et al. 1997a, 1997b, 1997c; Peterson \& Solensky 1988; Söderhjelm 1999).

Perryman et al. (1998), Lebreton et al. (1997) and Cayrel de Strobel et al. (1997) analysed the observational HR diagram of the Hyades with stellar models and obtained new estimates of the age, initial helium content and metallicity of the cluster (see Sect. 2.1). Recently, de Bruijne et al. (2000) and Castellani et al. (2000) compared the observational HR diagram with models and discussed the different uncertainties, in particular the problems related to the color-magnitude calibrations.

In this paper, we focus on the determination of the helium abundance of the Hyades through the analysis of both the M-L relation and HR diagram. We show that the excellent accuracies on the masses and luminosities reached for the Hyades binaries provide rather severe constraints on the helium content of the cluster and allow confirming and refining of the helium value previously inferred from the analysis of the HR diagram. In a second step, we discuss our ability to reproduce the various features of the HR diagram cluster sequence with different model input physics.

Section 2 is a brief review of previous studies of the observed HR diagram of the Hyades. Section 3 presents the observed Hyades M-L relation. Numerical stellar models are presented in Sect. 4. In Sect. 5, we analyse the M-L relation by means of stellar models and discuss the implications for the chemical composition of the cluster. In Sect. 6, we analyse and discuss the HR diagram of the cluster on the basis of the constraints provided by the M-L relation. Summary and conclusions are given in Sect. 7 .

\section{HR diagram data and previous analyses}

\subsection{Observational data}

Hipparcos measured the positions, proper motions and trigonometric parallaxes of $\sim 300$ candidate Hyades members allowing new, more detailed studies of the cluster to be undertaken.

Perryman et al. (1998) assigned the membership of 218 cluster stars and from the individual trigonometric parallaxes of the 134 stars located within $10 \mathrm{pc}$ of the cluster center, obtained a distance modulus $(m-M)=$ $3.33 \pm 0.01$ mag. Narayanan \& Gould (1999) used the Hipparcos proper motions to derive statistical parallaxes for 43 cluster stars leading to $(m-M)=3.34 \pm 0.02$ mag.

Dravins et al. (1997), and more recently de Bruijne et al. (2000), derived the dynamical parallaxes of $\sim 200$ Hyades members from the relation between the cluster space motion, the positions and the projected proper motions. As shown in Fig. 1, the two groups, who used the same data and similar methods, obtain parallaxes in excellent agreement; the dynamical parallaxes of the stars they have in common agree to better than 0.4 percent (except for one star, HIP 28356, where the parallax difference is about 1 percent). The mean accuracy on the dynamical

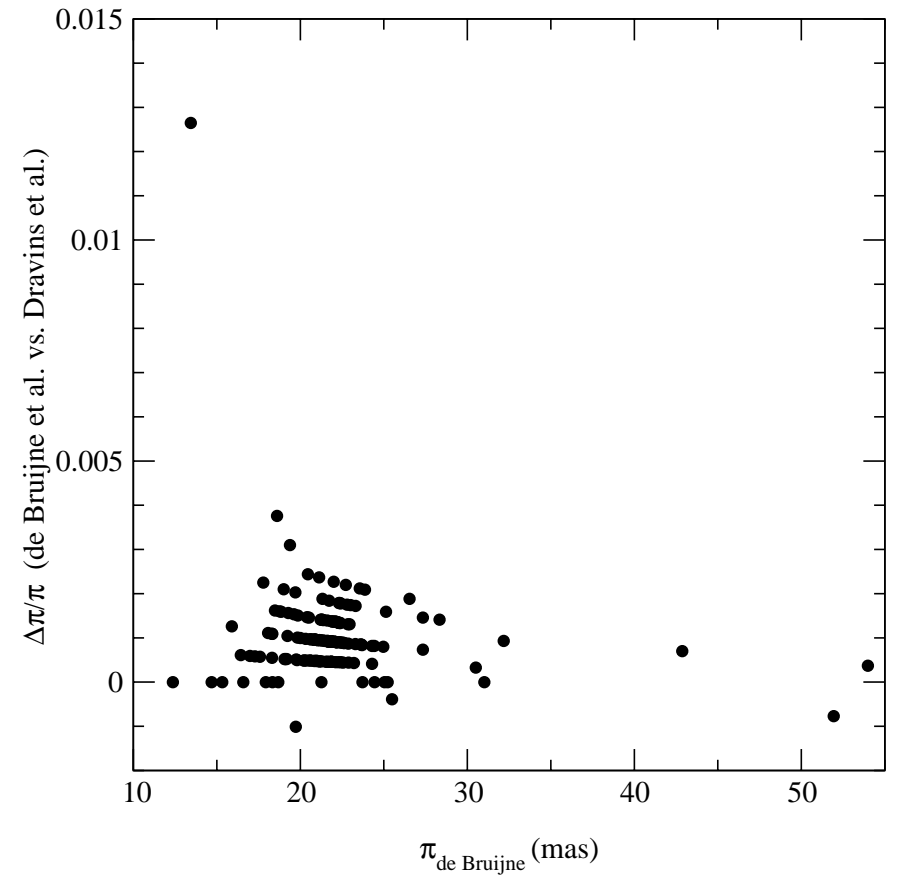

Fig. 1. Comparison of dynamical parallaxes of Hyades' stars as determined by de Bruijne et al. (2000) and by Dravins et al. (1997): all stars but one (HIP 28356) have their parallaxes within less than 0.4 percent.

parallaxes is $\sim 0.5$ mas, that is $\sim 3$ times better than the trigonometric parallax accuracy.

Photometric information can be found in the Hipparcos catalogue. The Hipparcos $H p$-magnitude of each observed star is given with an accuracy of $0.0015 \mathrm{mag}$. The $V$-magnitude and $(B-V)$ index of each star coming from ground-based measurements are also given in the catalogue, the typical accuracy on $V$ is better than $0.01 \mathrm{mag}$.

The mean absolute magnitude accuracy of the Hyades stars based on the dynamical parallaxes of Dravins et al. (1997) or of de Bruijne et al. (2000) is $\sigma_{M_{V}} \simeq 0.05$ mag. De Bruijne et al. (2000) examined 218 Hyades candidates among which they selected 90 secure Hyades members. The resulting $\left(M_{V}, B-V\right)$ colour-absolute magnitude diagram shows a very well-defined MS (i.e. $\left\langle\sigma_{M_{V}}\right\rangle \simeq 0.05 \mathrm{mag}$ and $\left\langle\sigma_{(B-V)}\right\rangle \simeq 0.01 \mathrm{mag}$, see Fig. 5).

The analysis of high-resolution, high signal-to-noise spectra of several Hyades stars has provided precise determinations of their effective temperatures, $T_{\text {eff }}$, and metallicities, $[\mathrm{Fe} / \mathrm{H}]$. Cayrel de Strobel et al. (1997) selected 40 Hyades dwarfs with $T_{\text {eff }}$ accurate to typically $50-70 \mathrm{~K}$; their mean metallicity $[\mathrm{Fe} / \mathrm{H}]=+0.14 \pm$ $0.05 \mathrm{dex}$, is in good agreement with the photometric value $[\mathrm{Fe} / \mathrm{H}]=+0.144 \pm 0.013$ dex derived by Grenon (2000) from large sets of homogeneous observations in the Geneva photometric system. 


\subsection{Previous analyses by means of stellar models}

Cayrel de Strobel et al. (1997) obtained the lower part of the cluster MS, in the $\left(M_{\mathrm{bol}}, T_{\mathrm{eff}}\right)$ plane, from the positions of 40 dwarfs, by combining Hipparcos distances, spectroscopic $T_{\text {eff }}, V$-magnitudes from the Hipparcos input catalogue and bolometric corrections by Bessel et al. (1998). Lebreton et al. (1997) and Perryman et al. (1998) compared the bottom of this sequence, corresponding to the non-evolved stars, with theoretical zero-age main sequences (ZAMS) computed with a $Z / X$ ratio of 0.034 corresponding to the mean observed $[\mathrm{Fe} / \mathrm{H}]$-value ${ }^{1}$. The ZAMS fitting yielded the initial helium content $Y=0.26 \pm$ 0.02 (in mass fraction) and metallicity $Z=0.024 \pm 0.004$ of the cluster, the (quite large) error on $Y$ being dominated by the error on the mean value of $[\mathrm{Fe} / \mathrm{H}]$ used $([\mathrm{Fe} / \mathrm{H}]=+0.14 \pm 0.05$ dex from spectroscopy $)$.

The position of the whole cluster sequence in the $\left(M_{V}\right.$, $B-V$ ) plane was obtained by Perryman et al. (1998) from the combination of Hipparcos distances and ground-based $V$-magnitudes and $(B-V)$ color indexes. The fitting of the sequence by model isochrones corresponding to the chemical composition of the cluster $(Y=0.26, Z=0.024)$ yielded an estimate of the age, $t \simeq 625 \mathrm{Myr}$.

In order to obtain a MS as fine as possible, the known or suspected unresolved binaries were removed from the samples mentioned above and the masses of the few resolved binaries were not used as constraints. Binaries with well-determined masses provide valuable constraints for stellar models, in particular for the stellar abundances through the M-L relation (see e.g. Andersen 1991). Some difficulties in fitting parts of the empirical Hyades M-L relation with models have been encountered by Torres et al. (1997b) and Lastennet et al. (1999), but the models (available from the literature) did not correspond to the cluster chemical composition. We now turn to the sample of binaries well-observed in the Hyades.

\section{Updated data for five binary systems}

We focus on five Hyades binary systems with welldetermined masses. The data are listed in Table 1. The dynamical parallaxes, $\pi_{\text {dyn }}$, are more precise than the direct Hipparcos trigonometrical parallaxes, $\pi_{\text {trig }}$ (Perryman et al. 1998). Therefore, in the following, we have used the dynamical parallaxes of de Bruijne et al. (2000) available for the five systems. We also list in Table 1 the $\pi_{\mathrm{dyn}}$-values derived by Dravins et al. (1997) in their improved version (Lindegren 1999, private communication).

For three systems (51 Tauri, Finsen 342 and $\theta^{2}$ Tau), which are double-lined spectroscopic binaries as well as visual binaries resolved by speckle, Torres et al. (1997a, 1997b, 1997c) derived a complete astrometricspectroscopic orbital solution, and therefore could obtain

\footnotetext{
${ }^{1} Z$ and $X$ are respectively the abundances in mass of heavy elements and hydrogen related to $[\mathrm{Fe} / \mathrm{H}]$ through $[\mathrm{Fe} / \mathrm{H}]=\log (Z / X)-\log (Z / X) \odot$ where $(Z / X)_{\odot}=0.0245$ according to Grevesse \& Noels (1993).
}

the individual masses and the orbital parallax, $\pi_{\text {orb }}$, listed in Table 1. 51 Tauri is also one of the 25 binary systems for which Söderhjelm (1999) could derive the individual masses combining Hipparcos data with ground-based observations. The resulting masses, $M_{\mathrm{A}}=1.72 \pm 0.27 M_{\odot}$ for the primary and $M_{\mathrm{B}}=1.31 \pm 0.21 M_{\odot}$ for the secondary are in good agreement with those obtained by Torres et al. Also, Martin et al. (1998) obtained the masses of the two components of 51 Tau from the analysis of Hipparcos data but with a lower accuracy $\left(M_{\mathrm{A}}=1.756 \pm 0.343 M_{\odot}\right.$ and $\left.M_{\mathrm{B}}=0.953 \pm 0.247 M_{\odot}\right)$.

For the $\theta^{1}$ Tau system, a single-lined spectroscopic binary with astrometric information from speckle and lunar occultation measurements, Torres et al. (1997c) obtained a partial astrometric-spectroscopic solution where the information coming from the velocity amplitude of the Bcomponent was lacking. However, it is possible to estimate the individual masses, using the parallax obtained independently from Hipparcos data. We give in Table 1 the values of the masses of the components of $\theta^{1}$ Tau derived from formulae (2), in Torres et al. (1997c), using their orbital parameters together with the Hipparcos $\pi_{\mathrm{dyn}, 2} \mathrm{dy}-$ namical parallax. It is worth noting that $\theta^{1}$ Tau $B$ and $\phi 342 \mathrm{~B}$ have very similar masses and magnitudes.

The fifth system, vB22, is a double-lined spectroscopic eclipsing binary discovered by McClure (1982). Individual masses were derived by Peterson \& Solensky (1988) and the radii were determined by Schiller \& Milone (1987).

The $V$-magnitude of each system, the magnitude difference between the two components $\Delta V$ and the corresponding individual absolute magnitudes $M_{V, \mathrm{~A}}$ and $M_{V, \mathrm{~B}}$

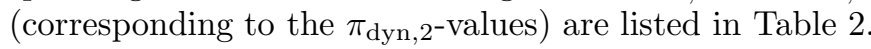
The individual values of the $(B-V)$ color index of the two components of vB22 have been determined by Schiller \& Milone (1987). For the other systems we infer the individual values of $(B-V)$ from the global value of the system given in WEBDA (http://obswww.unige.ch/webda/, see Mermilliod 1998) and from the difference $\Delta(B-V)$. For $\theta^{2}$ Tau, $\Delta(B-V)$ has been measured by Peterson et al. (1993). For 51 Tau and $\phi 342$, we follow Torres et al. (1997a) who derived $\Delta(B-V)$ from the value of $\Delta V$ and of the local slope of the empirical $(V, B-V)$ relation of Schwan et al. (1991) for the Hyades main sequence. For the $\theta^{1}$ Tau system, which is composed of a giant and of a dwarf, we only know the system $(B-V)$-value.

\section{Stellar models and related input physics}

We have calculated stellar models in the mass range $M \in[0.5,3.0] M_{\odot}$, using the CESAM code (Morel 1997) and the input physics described below.

1. Equation of state (EOS): the CEFF EOS (Eggleton et al. 1973, Christensen-Dalsgaard 1991) has mostly been used; it includes the Debye-Hückel corrections for pressure. The OPAL EOS (Rogers et al. 1996) that includes collective effects has been used at low mass for the purpose of comparison. 
Table 1. Parallaxes and masses for the 5 binary systems. The dynamical parallaxes $\pi_{\mathrm{dyn}, 1}$ are from Lindegren (1999, private communication but see also Dravins et al. 1997). The dynamical parallaxes $\pi_{\text {dyn,2 }}$ are from de Bruijne et al. (2000). The Hipparcos trigonometrical parallaxes $\pi_{\text {trig }}$ come from Perryman et al. (1998). As discussed in the text, the orbital parallaxes $\pi_{\text {orb }}$ and individual masses were obtained by Peterson \& Solensky (1988) for vB22 and by Torres et al. (1997a, 1997b, 1997c) for the other systems. The radii of the vB22 components are from Schiller \& Milone (1987) where the probable error has been translated into a mean error.

\begin{tabular}{|c|c|c|c|c|c|c|c|c|}
\hline name & HIP & $\mathrm{HD}$ & $\begin{array}{l}\pi_{\mathrm{dyn}, 1} \\
(\mathrm{mas})\end{array}$ & $\begin{array}{l}\pi_{\mathrm{dyn}, 2} \\
(\mathrm{mas})\end{array}$ & $\begin{array}{c}\pi_{\text {trig }} \\
(\text { mas })\end{array}$ & $\begin{array}{c}\pi_{\text {orb }} \\
(\text { mas })\end{array}$ & $\begin{array}{l}M_{\mathrm{A}} / M_{\odot} \\
M_{\mathrm{B}} / M_{\odot}\end{array}$ & $\begin{array}{l}R_{\mathrm{A}} / R_{\odot} \\
R_{\mathrm{B}} / R_{\odot}\end{array}$ \\
\hline vB22 & 20019 & 27130 & $21.15 \pm 0.33$ & $21.16 \pm 0.38$ & $21.40 \pm 1.24$ & $19.8 \pm 0.4$ & $\begin{array}{l}1.072 \pm 0.010 \\
0.769 \pm 0.005\end{array}$ & $\begin{array}{l}0.905 \pm 0.029 \\
0.773 \pm 0.015\end{array}$ \\
\hline 51 Tau & 20087 & 27176 & $18.29 \pm 0.24$ & $18.31 \pm 0.69$ & $18.25 \pm 0.82$ & $17.9 \pm 0.6$ & $\begin{array}{l}1.80 \pm 0.13 \\
1.46 \pm 0.18\end{array}$ & \\
\hline$\phi 342$ & 20661 & 27991 & $21.27 \pm 0.33$ & $21.29 \pm 0.37$ & $21.47 \pm 0.97$ & $21.44 \pm 0.67$ & $\begin{array}{l}1.363 \pm 0.073 \\
1.253 \pm 0.075\end{array}$ & \\
\hline$\theta^{1} \mathrm{Tau}$ & 20885 & 28307 & - & $21.29 \pm 0.37$ & $20.66 \pm 0.85$ & - & $\begin{array}{l}2.77 \pm 0.50 \\
1.28 \pm 0.13\end{array}$ & \\
\hline$\theta^{2}$ Tau & 20894 & 28319 & $22.21 \pm 0.35$ & $22.24 \pm 0.36$ & $21.89 \pm 0.83$ & $21.22 \pm 0.76$ & $\begin{array}{l}2.42 \pm 0.30 \\
2.11 \pm 0.17\end{array}$ & \\
\hline
\end{tabular}

Table 2. Magnitudes and colour indices for the five binary systems.

\begin{tabular}{|c|c|c|c|c|c|c|}
\hline name & HIP & HD & $\begin{array}{c}V \\
\Delta V\end{array}$ & $\begin{array}{l}M_{V, \mathrm{~A}} \\
M_{V, \mathrm{~B}}\end{array}$ & $\begin{array}{c}B-V \\
\Delta(B-V)\end{array}$ & $\begin{array}{l}(B-V)_{\mathrm{A}} \\
(B-V)_{\mathrm{B}}\end{array}$ \\
\hline vB22 & 20019 & 27130 & $\begin{array}{c}8.319 \pm 0.009 \\
2.3 \pm 0.05\end{array}$ & $\begin{array}{l}5.07 \pm 0.04 \\
7.37 \pm 0.06\end{array}$ & $\begin{array}{l}- \\
-\end{array}$ & $\begin{aligned} 0.713 & \pm 0.017 \\
1.19 & \pm 0.08\end{aligned}$ \\
\hline 51 Tau & 20087 & 27176 & $\begin{array}{l}5.65 \pm 0.01 \\
1.61 \pm 0.10\end{array}$ & $\begin{array}{l}2.18 \pm 0.09 \\
3.79 \pm 0.12\end{array}$ & $\begin{array}{l}0.28 \pm 0.01 \\
0.19 \pm 0.01\end{array}$ & $\begin{array}{l}0.25 \pm 0.05 \\
0.44 \pm 0.02\end{array}$ \\
\hline$\phi 342$ & 20661 & 27991 & $\begin{array}{l}6.46 \pm 0.01 \\
0.34 \pm 0.05\end{array}$ & $\begin{array}{l}3.70 \pm 0.05 \\
4.03 \pm 0.05\end{array}$ & $\begin{array}{l}0.49 \pm 0.01 \\
0.05 \pm 0.01\end{array}$ & $\begin{array}{l}0.466 \pm 0.056 \\
0.530 \pm 0.020\end{array}$ \\
\hline$\theta^{1}$ Tau & 20885 & 28307 & $\begin{array}{l}3.84 \pm 0.01 \\
3.50 \pm 0.05\end{array}$ & $\begin{array}{l}0.52 \pm 0.04 \\
4.02 \pm 0.07\end{array}$ & $\begin{array}{c}0.95 \pm 0.01 \\
-\end{array}$ & $\begin{array}{l}- \\
-\end{array}$ \\
\hline$\theta^{2}$ Tau & 20894 & 28319 & $\begin{array}{l}3.40 \pm 0.02 \\
1.10 \pm 0.01\end{array}$ & $\begin{array}{l}0.48 \pm 0.04 \\
1.58 \pm 0.05\end{array}$ & $\begin{array}{c}0.18 \pm 0.01 \\
-0.006 \pm 0.005\end{array}$ & $\begin{array}{l}0.18 \pm 0.02 \\
0.17 \pm 0.02\end{array}$ \\
\hline
\end{tabular}

2. Opacities: we used OPAL (Iglesias \& Rogers 1996) complemented by Alexander \& Ferguson's (1994) data for $T \lesssim 10^{4} \mathrm{~K}$, both being for Grevesse \& Noels's (1993) solar mixture.

3. Thermonuclear reaction rates: Caughlan \& Fowler (1988).

4. Standard mixing-length theory of convection (BöhmVitense 1958). The calibration of the solar model in luminosity and radius with a particular set of input physics yields the solar mixing-length ratio $\alpha_{\mathrm{MLT}, \odot}=$ $l / H_{\mathrm{p}} \quad\left(H_{\mathrm{p}}\right.$ is the pressure scale-height). The value of $\alpha_{\mathrm{MLT}, \odot}$ depends on the external boundary conditions adopted. We find $\alpha_{\mathrm{MLT}, \odot}=1.61$ for the Eddington's grey model atmosphere and $\alpha_{\mathrm{MLT}, \odot}=$ 1.79 for Kurucz's (1991) ATLAS9 models (see item 7). Models calculated with $\alpha_{\mathrm{MLT}, \odot}$ also give a good fit of the MS slope of the nearby stars (Lebreton et al. 1999) and of the Hyades (Perryman et al. 1998). In addition, the calibration in luminosity and effective temperature of several nearby visual binaries with determined masses yields values of $\alpha_{\mathrm{MLT}}$ close to $\alpha_{\mathrm{MLT}, \odot}$ (Fernandes et al. 1998). From numerical 2-D simulations of convection in a wide range of $T_{\text {eff }}$ and $\log g$
( $g$ is the surface gravity), Ludwig et al. (1999) obtained equivalent $\alpha_{\mathrm{MLT}}$-values in the range 1.1-1.8 for solar or subsolar $[\mathrm{Fe} / \mathrm{H}]$ - they did not investigate supersolar metallicites as found in the Hyades. For low mass $M S$ stars these simulations suggest possible variations of $\alpha_{\mathrm{MLT}}$ with $T_{\text {eff }}$ and log $g$ in the range $\alpha_{\mathrm{MLT}, \odot} \pm 0.15$. We therefore adopted the solar mixing-length ratio for standard models but we discuss the effects of a change of $\alpha_{\mathrm{MLT}}$ as high as \pm 0.40 .

5. Convective core overshooting. We take $d_{\mathrm{ov}}=0.2 H_{\mathrm{p}}$ as overshooting distance, a value derived by Schaller et al. (1992) from the study of the empirical MS width of open clusters. We also discuss the case with no overshooting.

6. Microscopic diffusion transports helium and heavy elements toward the center of stars and may change their surface and core compositions during evolution. It can be important in rather old metal-poor stars (see e.g. Proffitt \& Vandenberg 1991). We calculated models including microscopic diffusion and checked that in the Hyades, which are rather young metal-rich objects, the effects of microscopic diffusion are small enough to be neglected in the present study. 
7. External boundary conditions. The $T(\tau)$-law is taken from the classical Eddington grey model atmosphere except in a few models where we used, for comparison, a $T(\tau)$-law derived from Kurucz's (1991) complete ATLAS9 model atmospheres.

To fit the Hyades empirical M-L relation and HR diagram, we calculated evolution models and isochrones with various values of the initial helium abundance $Y$ and metallicity $Z / X$. The $Z / X$-ratio can vary in the range $0.034 \pm 0.005$ given by the observed $[\mathrm{Fe} / \mathrm{H}]$-value (see Sect. 2). The error bar on $Z / X(0.005)$ includes the 0.05 dex error on $[\mathrm{Fe} / \mathrm{H}]$ and the 11 per cent error on $(Z / X)_{\odot}$ quoted by Anders \& Grevesse (1989). The helium mass fraction $Y$ is allowed to vary in the range $0.235-$ 0.30 , i.e. from the primordial abundance (see Peimbert et al. 2000) to the approximative upper limit given by observations (see Nissen 1976). In the following, we refer to solar-scaled helium when the helium abundance is related to metallicity through the well-known relation $\left(Y-Y_{\mathrm{p}}\right) / Z=\Delta Y / \Delta Z=(\Delta Y / \Delta Z)_{\odot}$ where $Y_{\mathrm{p}}=0.235$ is the primordial helium value and $(\Delta Y / \Delta Z)_{\odot}$ is derived from the calibration in luminosity and radius of the solar model. For a solar model that does not take into account the microscopic diffusion we obtain $Y_{\odot}=0.2674$ and $Z_{\odot}=0.0175$ and in turn $(\Delta Y / \Delta Z)_{\odot}=1.9$.

The conversion of the theoretical model outputs $\left(M_{\mathrm{bol}}\right.$, $\left.T_{\text {eff }}\right)$ into the observational plane $\left(M_{V}, B-V\right)$ has been performed with the most recent version of the BaseL Stellar Library (BaSeL, version 2.2), available electronically at http://www.astro.mat.uc.pt/BaSeL/ (Lejeune 2001). BaSeL provides color-calibrated theoretical flux distributions for the largest possible range of fundamental stellar parameters, $T_{\text {eff }}(2000$ to $50000 \mathrm{~K}), \log g(-1.0$ to $5.5 \mathrm{dex})$, and $[\mathrm{Fe} / \mathrm{H}](-5.0$ to $+1.0 \mathrm{dex})$.

The BaSeL flux distributions are calibrated on the stellar UBVRIJHKL colors, using these empirical photometric calibrations for solar metallicity, and semi-empirical relations constructed from the color differences predicted by the stellar model atmospheres for non-solar metallicities (details about the calibration procedure are given in Lejeune et al. 1997, 1998).

We point out that although Hipparcos provided its own precise magnitudes $H p$ (see Sect. 2.1), it has not been possible to use them here because there are still problems in computing the bolometric corrections corresponding to the $\mathrm{Hp}$ band (Cayrel et al. 1997).

All the models and isochrones presented here can be sent on request.

\section{Constraints from the $M-L$ relation}

The vB22-components have the lowest and most accurate masses $\left(\sigma_{M} / M<1 \%\right)$ and define the lower part of the M$\mathrm{L}$ relation quite accurately. From the models we find that at 650 Myr (age of the Hyades, according to Perryman et al. 1998), both components are only slightly evolved,

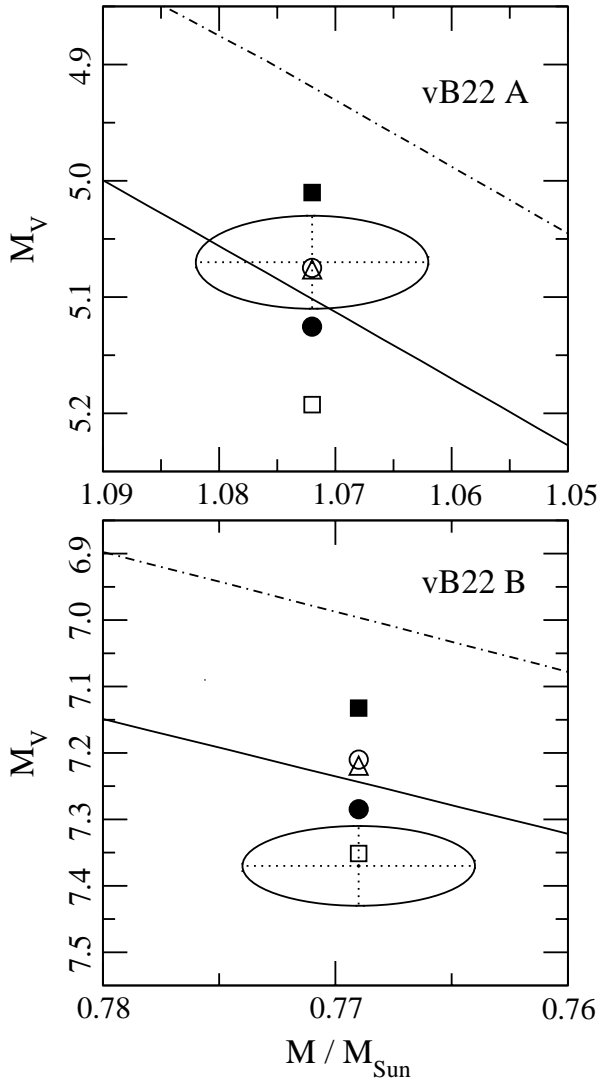

Fig. 2. The vB22 system in the M-L plane (data from Tables 1 and 2). The ellipses represent the locus where $\left(M_{V}-\right.$ $\left.M_{V, \text { obs }}\right)^{2} / \sigma_{M_{V}}^{2}+\left(M-M_{\text {obs }}\right)^{2} / \sigma_{M}^{2} \leq 1$. Models aged $625 \mathrm{Myr}$ are plotted. Continuous line: three indistinguishable isochrones with $([\mathrm{Fe} / \mathrm{H}], Y) \equiv(0.09,0.25),(0.14,0.26)$ and $(0.19,0.27)$. Dot-dashed line: isochrone with $[\mathrm{Fe} / \mathrm{H}]=0.14$ dex and solarscaled helium $Y=0.28$. Circles: models with $([\mathrm{Fe} / \mathrm{H}], Y) \equiv$ $(0.14,0.26)$ and $\alpha_{\mathrm{MLT}}=\alpha_{\mathrm{MLT}, \odot}-0.20(\bullet)$ or $\alpha_{\mathrm{MLT}, \odot}+0.20(\circ)$. Squares: $([\mathrm{Fe} / \mathrm{H}], Y) \equiv(0.09,0.24),(0.14,0.25),(0.19,0.26)$ $(\square) ;([\mathrm{Fe} / \mathrm{H}], Y) \equiv(0.09,0.26),(0.14,0.27),(0.19,0.28)(\boldsymbol{\square})$. Open triangle: $([\mathrm{Fe} / \mathrm{H}], Y) \equiv(0.14,0.26)$ and OPAL EOS. The smallness of the error bars allows us to discriminate between rather close values of $Y$ and to eliminate the isochrone with solar-scaled helium.

the departure from the ZAMS position being less than $0.06 \mathrm{mag}$ for $\mathrm{vB} 22 \mathrm{~A}$ (0.03 mag for vB22B).

The positions of the vB22 components in the M-L plane are plotted in Fig. 2 where we have superimposed models and isochrones aged $625 \mathrm{Myr}$. The first important conclusion that can be drawn is that (with the present set of models) the constraints imposed by the M-L relation are hardly compatible with a solar-scaled helium value: the $([\mathrm{Fe} / \mathrm{H}], Y) \equiv(0.14,0.28)$ isochrone lies more than $3 \sigma$ above the data. This possibility had already been suspected by Torres et al. (1997b). Even if we allow $[\mathrm{Fe} / \mathrm{H}]$ to vary inside its error bars, we find that an isochrone with $[\mathrm{Fe} / \mathrm{H}]=0.10$ dex and solar-scaled helium (not represented in the figure) sits more than $2 \sigma$ above the data. Errors on the bolometric corrections (BC) could be invoked but they should be quite high. The BaSeL transformations applied to $\left(T_{\text {eff }}, \log g\right)$-values close to 


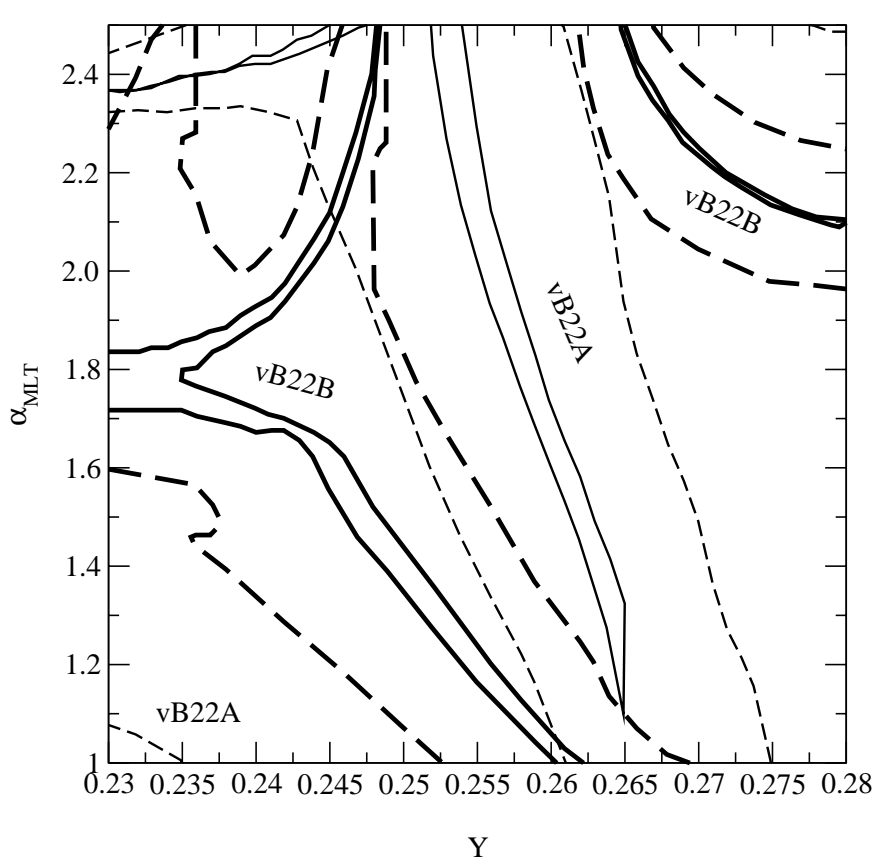

Fig. 3. Contour levels in the $Y$ - $\alpha_{\text {MLT }}$ plane. Continuous lines are for $\chi^{2}=\left(M_{V \text {, model }}-M_{V \text {,obs }}\right)^{2} /{\sigma_{M_{V}}}^{2}+\left(M_{\text {model }}-\right.$ $\left.M_{\text {obs }}\right)^{2} / \sigma_{M}{ }^{2}=10^{-2}$, dashed for $\chi^{2}=1$. vB22B is in bold lines.

those of the vB22 system lead to $\mathrm{BC}_{\mathrm{A}} \approx-0.1 \mathrm{mag}$ and $\mathrm{BC}_{\mathrm{B}} \approx-0.7 \mathrm{mag}$. On the other hand, for both stars, the BaSeL BCs do not differ by more than 0.03 mag from Alonso et al.'s (1995) empirical corrections. To reconcile models with solar-scaled helium with observations, the $\mathrm{BC}$ should be changed by $\triangle \mathrm{BC}_{\mathrm{A}} \sim 0.15 \mathrm{mag}$ and $\Delta \mathrm{BC}_{\mathrm{B}} \sim 0.40 \mathrm{mag}$, which appears to be very high. A helium content lower than the solar-scaled value is therefore favoured.

The well-known degeneracy between helium and metallicity in the HR and M-L diagrams makes isochrones with different $([\mathrm{Fe} / \mathrm{H}], \mathrm{Y})$ values coincide exactly. Models give $\partial M_{V} / \partial Y=-10 \pm 1$ and $\partial M_{V} / \partial[\mathrm{Fe} / \mathrm{H}]=2.0 \pm 0.2$. That means that a change in $Y$ of -0.01 is compensated for by an increase in $[\mathrm{Fe} / \mathrm{H}]$ by 0.05 dex.

Several error sources bring uncertainties in the $Y$ determination. The errors on mass and visual magnitude each lead to $\Delta Y \approx 0.005$. An error of $0.05 \mathrm{mag}$ on the bolometric correction also gives $\Delta Y \approx 0.005$. Depending on the choice made for the Hyades $[\mathrm{Fe} / \mathrm{H}]$-value (i.e. spectroscopic or photometric determination) the $[\mathrm{Fe} / \mathrm{H}]$ contribution to the $Y$-error budget will be dominant or not: spectroscopy gives $\Delta[\mathrm{Fe} / \mathrm{H}]=0.05$ dex and in turn $\Delta Y=0.01$ while photometry yields $\Delta[\mathrm{Fe} / \mathrm{H}]=0.013$ dex leading to $\Delta Y=0.0026$

We also investigated how the models change when the input physics or input parameters are modified:

- the component magnitudes are not changed by more than $0.001 \mathrm{mag}$ if microscopic diffusion or pre-main sequence evolution calculations are included,

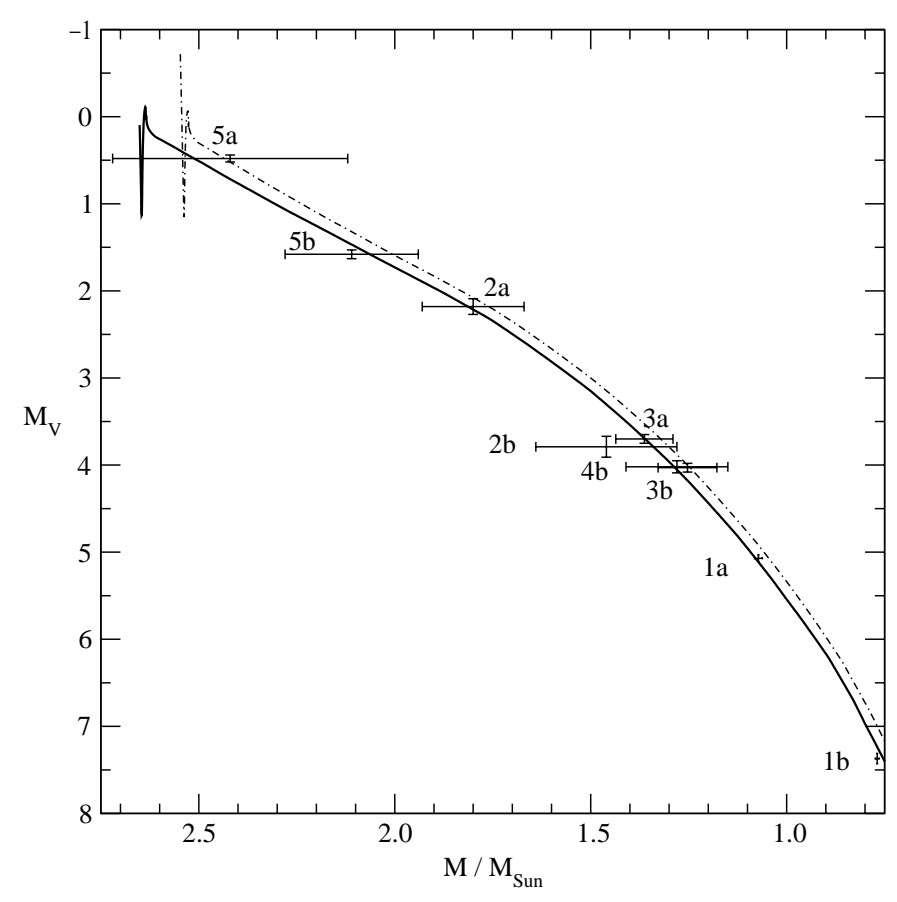

Fig. 4. The Hyades M-L relation for vB22 (1), 51 Tau (2), $\phi 342(3), \theta^{1}$ Tau (4) and $\theta^{2}$ Tau (5) and model isochrones aged 625 Myr. Continuous line: three indistinguishable isochrones with $([\mathrm{Fe} / \mathrm{H}], Y) \equiv(0.09,0.25),(0.14,0.26)$ and $(0.19,0.27)$. Dot-dash line: $[\mathrm{Fe} / \mathrm{H}]=0.14$ dex and solar-scaled helium $Y=$ 0.28 . Here the error bars on mass are too large to discriminate between various helium values (vB22 excepted).

- adopting the OPAL EOS instead of CEFF decreases both magnitudes by $0.02 \mathrm{mag}$,

- changing the Eddington's grey model atmosphere into Kurucz's model only modifies the vB22B magnitude (by $+0.03 \mathrm{mag}$ ),

- small age variations do not change the magnitudes much $\left(\partial M_{V} / \partial t \lesssim 10^{-4}\right.$ mag per Myr),

- varying $\alpha_{\mathrm{MLT}}$ around $\alpha_{\mathrm{MLT}, \odot}$ yields $\partial M_{V} / \partial \alpha_{\mathrm{MLT}}=$ 0.13 for $\mathrm{vB} 22 \mathrm{~A}(0.20$ for $\mathrm{vB} 22 \mathrm{~B})$.

Using the OPAL EOS, we varied $Y$ and $\alpha_{\mathrm{MLT}}$ to find models of vB22A and vB22B satisfying $\chi^{2}=\left(M_{V \text {, model }}-\right.$ $\left.M_{V \text {,obs }}\right)^{2} / \sigma_{M_{V}}{ }^{2}+\left(M_{\text {model }}-M_{\text {obs }}\right)^{2} / \sigma_{M^{2}} \leq 1$. We took account of an error on the bolometric corrections of $\Delta \mathrm{BC}=$ 0.05 mag. Figure 3 shows the $\chi^{2}$-contour levels in the $Y$ $\alpha_{\mathrm{MLT}}$ plane. If we force $\alpha_{\mathrm{MLT}}$ to be in the range $1.2-2.0$ (i.e. $\alpha_{\mathrm{MLT}}=\alpha_{\mathrm{MLT}, \odot} \pm 0.4$, see item 4 , Sect. 4 ) and if we require that the initial helium content be the same in the two stars, we find solutions for $Y$ in the range $0.247-0.263$. In that $Y$-range we can have $\alpha_{\mathrm{MLT}, \mathrm{A}}=\alpha_{\mathrm{MLT}, \mathrm{B}}$ but the smallest $\chi^{2}$ are obtained for $\Delta \alpha_{\mathrm{MLT}}=\alpha_{\mathrm{MLT}, \mathrm{A}}-\alpha_{\mathrm{MLT}, \mathrm{B}} \neq 0$ with a trend for $\Delta \alpha_{\mathrm{MLT}}$ to increase when $Y$ decreases.

In summary, the helium content of the Hyades deduced from models based on the OPAL EOS is $Y=0.255 \pm 0.009$ $([\mathrm{Fe} / \mathrm{H}]$ from photometry) or $Y=0.255 \pm 0.013([\mathrm{Fe} / \mathrm{H}]$ from spectroscopy), that we write

$Y=[0.255+0.2([\mathrm{Fe} / \mathrm{H}]-0.14)] \pm 0.008$ 
to highlight the effect of the $[\mathrm{Fe} / \mathrm{H}]$-uncertainty. This value is fully compatible with $Y=0.26 \pm 0.02$ derived by Perryman et al. (1998) from the model fit of 40 ZAMS stars with spectroscopic $T_{\text {eff }}$. The changes in the input physics considered here do not modify that result by more than $\Delta Y=0.002$. Present models also suggest that $\alpha_{\mathrm{MLT}}$ could be different in the two components, and higher in vB22A, but the error bars on observations are still too large to draw firm conclusions on that point.

As pointed out by Lastennet et al. (1999), models with solar-scaled helium and a solar mixing-length ratio cannot satisfy the mass-radius (M-R) and the M-L relation simultaneously. From our models we find that, quite independent of the helium abundance, only vB22A models with high $\alpha_{\mathrm{MLT}}\left(\alpha_{\mathrm{MLT}}>1.8\right)$ and vB22B models with very low $\alpha_{\text {MLT }}\left(\alpha_{\text {MLT }}<1.0\right)$ can give the observed radii. The simultaneous fit of the M-L and M-R relations is possible for vB22A but imposes hardly acceptable values of $\alpha_{\mathrm{MLT}}$ in vB22B. The use of Kurucz's model atmospheres to fix the external boundary conditions of the models does not modify this conclusion.

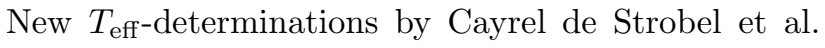
(2001) give $T_{\text {eff, }}=5600 \pm 75 \mathrm{~K}$ and $T_{\text {eff,B }}=4500 \pm 75 \mathrm{~K}$. The radii of $\mathrm{vB} 22 \mathrm{~A}$ and $\mathrm{vB} 22 \mathrm{~B}$ can be derived from the observed magnitudes and these $T_{\text {eff }}$ through StefanBoltzmann's law and using bolometric corrections from BaSeL. We find $R_{\mathrm{A}}=0.97 \pm 0.05 R_{\odot}$ and $R_{\mathrm{B}}=0.64 \pm$ $0.06 R_{\odot}$. The radii based on Stefan-Boltzmann's law are hardly compatible with and much less precise than the radii inferred from observed eclipses (see Table 1). A higher $R_{\mathrm{A}}$ (smaller $R_{\mathrm{B}}$ ) implies a smaller $\alpha_{\mathrm{MLT}, \mathrm{A}}$ (higher $\left.\alpha_{\mathrm{MLT}, \mathrm{B}}\right)$ and therefore it is easier to find models with $\alpha_{\mathrm{MLT}}$ in the range $\alpha_{\mathrm{MLT}, \odot} \pm 0.4$ that fit both the M-L relation and radii from Stefan-Boltzmann's law. To draw definite conclusions on the validity of the models, it is important to rediscuss the observations thoroughly. In particular, the radii from eclipses should be firmly assessed because they are given with such high precision that they strongly constrain the models.

Figure 4 shows the whole M-L relation for the 5 binary systems. Because the error bars on the component masses are quite large (i.e. in the range 5-25 percents), no additional constraint on the helium abundance comes from the four systems $\phi 342,51 \mathrm{Tau}, \theta^{1}$ and $\theta^{2}$ Tau. In particular, $\theta^{2}$ Tau A lies in the hook region (end of the core H-burning phase) but its exact position on the hook cannot be determined because of its large mass error bar.

\section{Revisited analysis of the HR diagram}

Figure 5 shows the colour-magnitude diagram of 90 secure Hyades members (de Bruijne et al. 2000) complemented by the data of Table 2 for four of the binary systems examined in Sect. 3 above $\left(\theta^{1}\right.$ Tau has been omitted because no reliable information on the individual $(B-V)$-values is available). A model isochrone, aged $650 \mathrm{Myr}$, calculated with the metallicity of the Hyades $([\mathrm{Fe} / \mathrm{H}]=0.14 \mathrm{dex})$ and

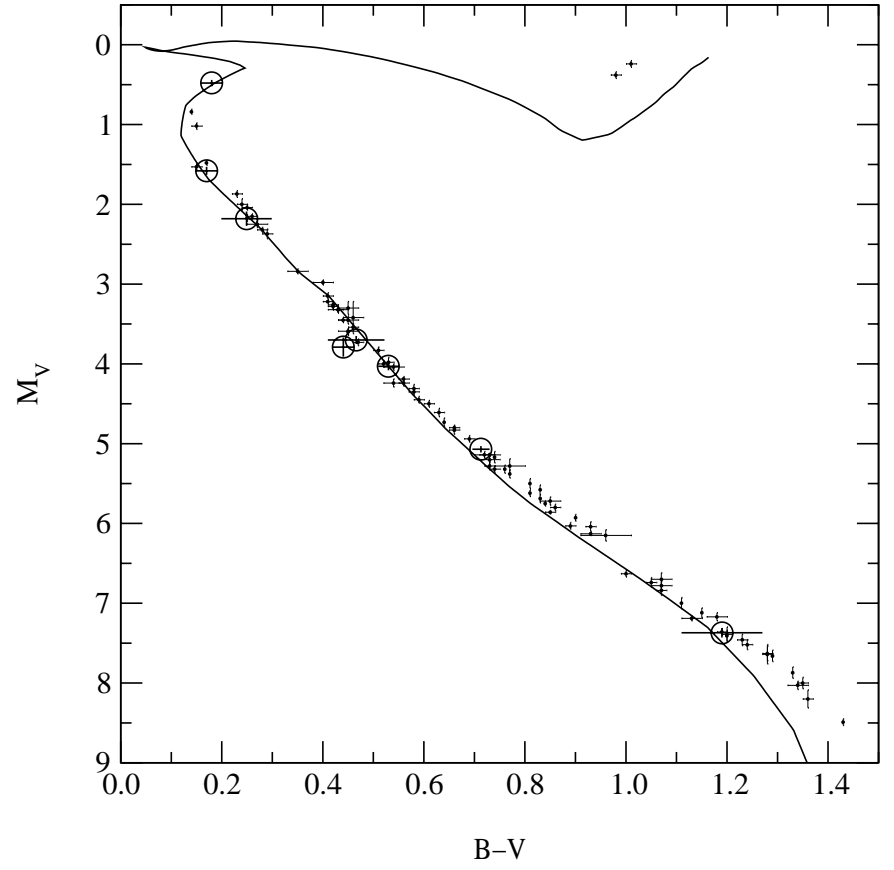

Fig. 5. Hyades's HR diagram. Data are from de Bruijne et al. (2000) complemented by the data of Table 2 for four of the binary systems (o). Isochrone: $650 \mathrm{Myr}, Y=0.26,[\mathrm{Fe} / \mathrm{H}]=0.14$, $\alpha_{\mathrm{MLT}}=\alpha_{\mathrm{MLT}, \odot} \cdot$

a helium abundance fixed by the M-L relation $(Y=0.26)$ is also plotted in Fig. 5 showing that:

1. In the range $(B-V) \in[0.3,1.15]$ the fit of the data is quite good, although for $(B-V) \in[0.7,0.9]$ some stars sit above the isochrone;

2. For $(B-V) \gtrsim 1.15$ the isochrone is much too blue with respect to the data: there is a change of slope at low mass both in the data and in the isochrone but it occurs at lower $(B-V)$ in the isochrone;

3. For $(B-V) \lesssim 0.3$, corresponding to the turn-off region, a few stars are located to the right of the isochrone.

Figure 6 shows the same data with, in addition, 54 known (unresolved) binaries with dynamical parallaxes from Dravins et al. (1997). It shows that, except at low mass $((B-V) \gtrsim 1.15)$ where the slope of the isochrone is bad, the binaries sit well in the $\Delta M_{V} \sim 0.75$ mag band, where they are expected to be.

In the following, we shall only discuss the MS and turnoff regions. The isochrones presented here do not go far enough to cover the giants, in the He-burning stage. In this region, only two secure members are found, plus $\theta^{1}$ Tau A. For a discussion of the constraints associated with the giant region, see de Bruijne et al. (2000) and Castellani et al. (2000).

\subsection{Helium abundance}

Figure 7 illustrates the differences between two isochrones of same age and same metallicity $([\mathrm{Fe} / \mathrm{H}]=0.14)$ but different helium abundances $(Y=0.26$ and solar-scaled 


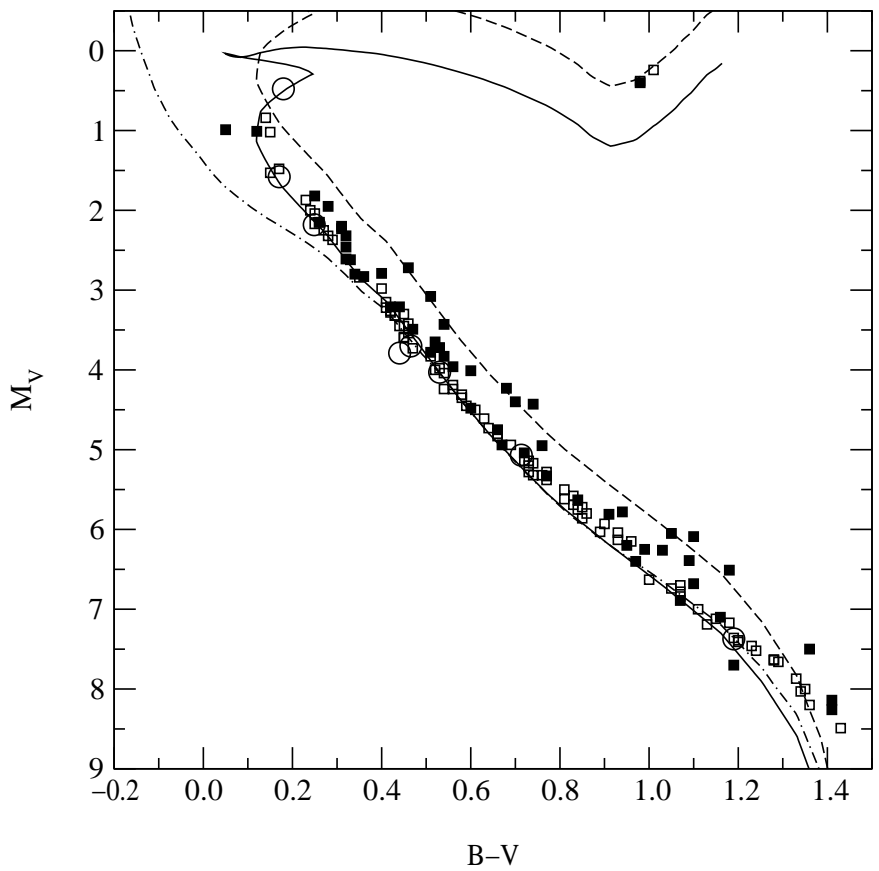

Fig. 6. The HR diagram including unresolved known binaries. Open squares are data for single stars from de Bruijne et al. (2000), open circles are for the four resolved binary systems and full squares are the unresolved binaries with dynamical parallaxes from Dravins et al. (1997). The continuous line is the $(650 \mathrm{Myr}, Y=0.26,[\mathrm{Fe} / \mathrm{H}]=0.14)$-isochrone, dashed line is the same isochrone translated by $-2.5 \log 2$ in $M_{V}$ giving the maximum shift expected from unresolved binaries. The dotdashed isochrone is 50 Myr old.

$Y=0.28)$. The isochrone that gives a better fit to the M-L relation (i.e. $Y=0.26$ ) also provides a better fit to the low MS. However the constraints in the $\left(M_{V}, B-V\right)$ plane are not so strong (the difference in $B-V$ between the two isochrones are lower than the mean error bar on $B-V)$.

Like Castellani et al. (2000), we find that the isochrone with solar scaled helium departs from the data as soon as $(B-V) \gtrsim 0.6$. Castellani et al. suggest that a decrease of $\alpha_{\mathrm{MLT}}$ could improve the fit at least for $(B-V) \in[0.7,1.1]$. According to their Fig. 2 a rather large decrease of $\alpha_{\mathrm{MLT}}$ would be necessary (by about $\sim 0.4$ ). We discuss $\alpha_{\mathrm{MLT}}$ changes in Sect. 6.3.

\subsection{Metallicity}

Figure 8 shows two isochrones with extreme $[\mathrm{Fe} / \mathrm{H}]$-values (0.09 and 0.19) that were indistinguishable in the M-L plane (Sect. 5). In the colour magnitude diagram they differ, but by a very small amount. On the MS, a variation of 0.05 dex in $[\mathrm{Fe} / \mathrm{H}]$ changes $(B-V)$ by less than $0.01 \mathrm{mag}$. The present data have $\left\langle\sigma_{(B-V)}\right\rangle \simeq 0.01$ mag which hardly allows to discriminate between $[\mathrm{Fe} / \mathrm{H}]$-values inside the observed range, $\Delta[\mathrm{Fe} / \mathrm{H}]=0.013 \mathrm{dex}(0.05)$ from photometry (spectroscopy). On the other hand, an uncertainty of 0.05 dex on $[\mathrm{Fe} / \mathrm{H}]$ gives a $25 \mathrm{Myr}$ uncertainty on the age.

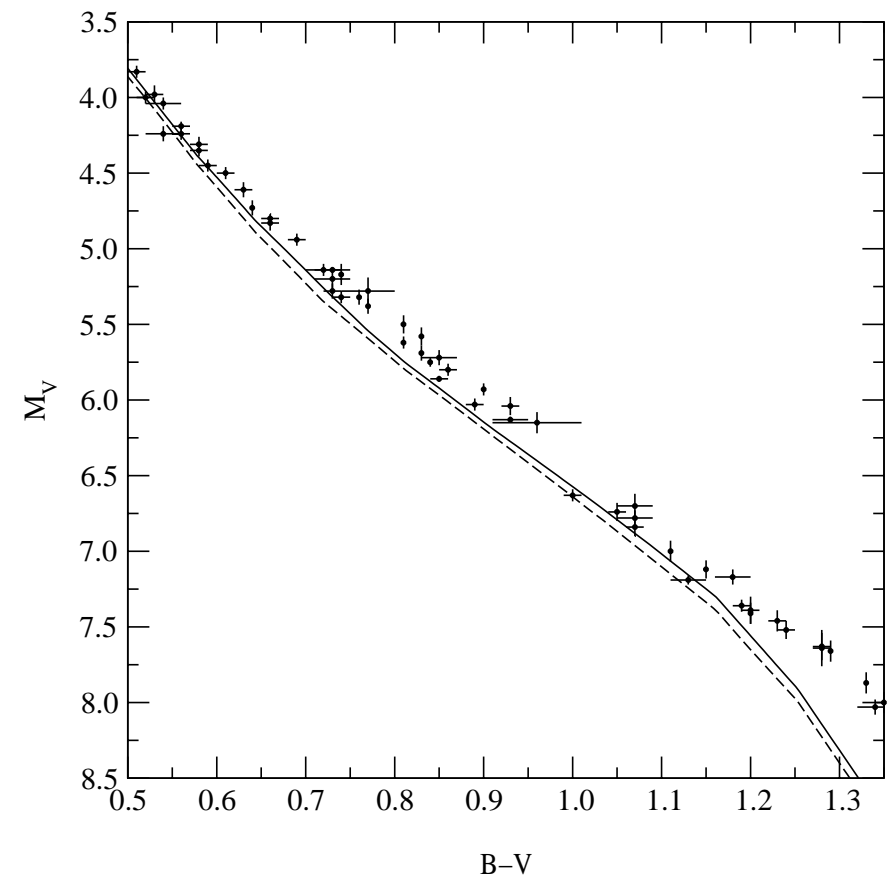

Fig. 7. Helium: zoom on the non-evolved region. Isochrones aged $650 \mathrm{Myr}$, with $[\mathrm{Fe} / \mathrm{H}]=0.14$ and $Y=0.26$ (continuous line) or solar-scaled helium $Y=0.28$ (dash).

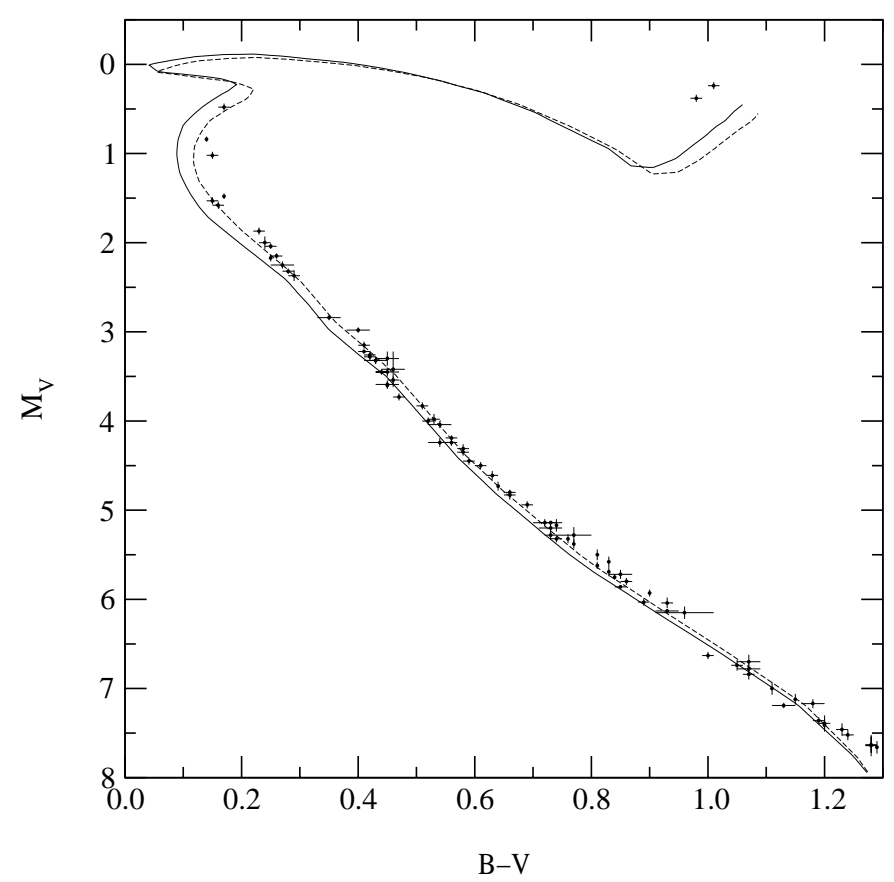

Fig. 8. Two isochrones aged $650 \mathrm{Myr}$ with "extreme" $[\mathrm{Fe} / \mathrm{H}]-$ values are plotted: the continuous line is for $([\mathrm{Fe} / \mathrm{H}]=0.09$, $Y=0.25)$, dashed for $([\mathrm{Fe} / \mathrm{H}]=0.19, Y=0.27)$.

\subsection{MS slope}

The effects of a change in $\alpha_{\mathrm{MLT}}$ on the $(B-V)$ colour index are plotted in Fig. 9 as a function of $M_{V}$ for different values of metallicity (metal-poor $[\mathrm{Fe} / \mathrm{H}]=-1.0$, solar $[\mathrm{Fe} / \mathrm{H}]=0.0$ and Hyades $[\mathrm{Fe} / \mathrm{H}]=+0.14 \mathrm{dex})$. We considered a \pm 0.20 deviation of $\alpha_{\mathrm{MLT}}$ from the 
solar value $\left(\alpha_{\mathrm{MLT}, \odot} \sim 1.6\right)$. At any metallicity a significant shift in $(B-V)$ is expected for magnitudes in the range 4.-7. mag, with a maximum shift at $M_{V} \simeq 5.0-5.5 \mathrm{mag}$. The higher the metallicity, the higher the $(B-V)$ shift. At Hyades metallicity, the $(B-V)$ shift is in the range $\sim 0.01-0.03$ mag. This effect is of the order of, and even larger than, the mean error bar on $(B-V)$ for the Hyades data $\left(\left\langle\sigma_{(B-V)}\right\rangle \simeq 0.01 \mathrm{mag}\right)$.

Figure 10 is a zoom of the Hyades MS over the magnitude range where the $\alpha_{\mathrm{MLT}}$ changes have the largest effects. Four isochrones with $\alpha_{\mathrm{MLT}}=\alpha_{\mathrm{MLT}, \odot}-0.40$, $\alpha_{\mathrm{MLT}, \odot}-0.20, \alpha_{\mathrm{MLT}, \odot}$, and $\alpha_{\mathrm{MLT}, \odot}+0.20$ are superimposed on the data. All stars with $(B-V) \in[0.4,1.1]$ lie in the band where $\alpha_{\mathrm{MLT}, \odot}-0.4<\alpha_{\mathrm{MLT}}<\alpha_{\mathrm{MLT}, \odot}+0.2$. With the present set of model isochrones, we note a trend for stars of decreasing mass to lie on isochrones having decreasing $\alpha_{\mathrm{MLT}}$-values. This suggests $\alpha_{\mathrm{MLT}}$-values in the range $\sim 1.6-1.8$ for $M \sim 1.3-1.7 M_{\odot}, \alpha_{\mathrm{MLT}} \approx 1.4-1.6$ for $M \sim 1.0-1.3 M_{\odot}$ and $\alpha_{\mathrm{MLT}} \lesssim 1.4$ below $1.0 M_{\odot}$.

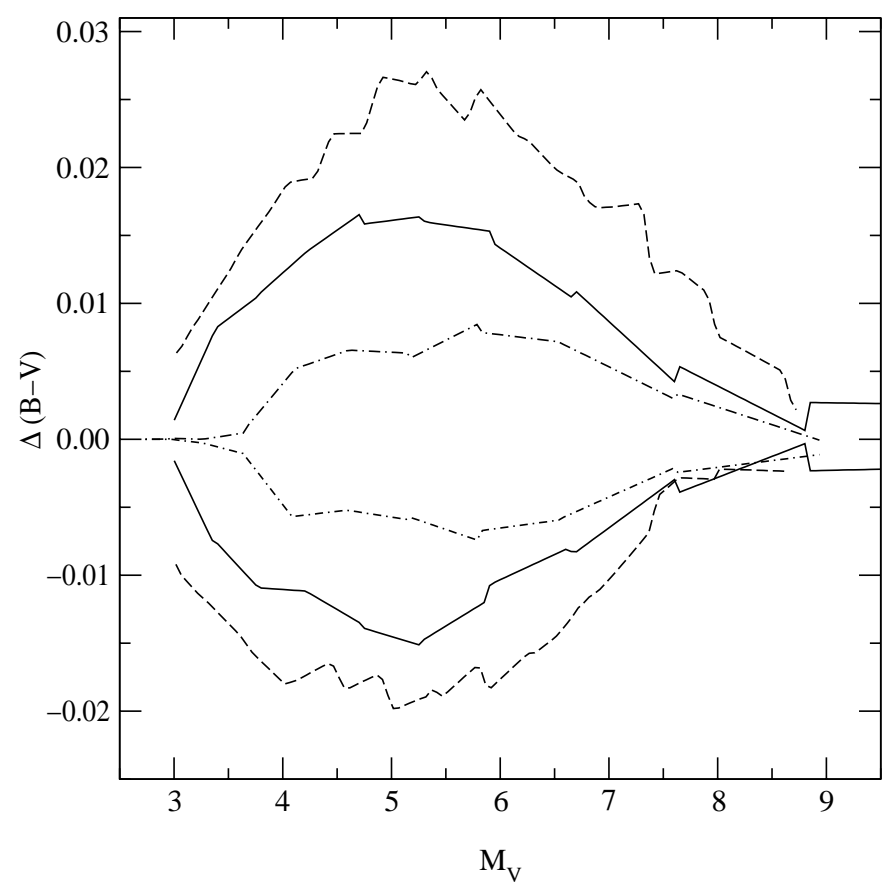

Fig. 9. Effect of a change of $\alpha_{\mathrm{MLT}}$ on the MS position. $\Delta(B-$ $V)$ is the shift at constant magnitude between an isochrone with $\alpha_{\mathrm{MLT}}=\alpha_{\mathrm{MLT}, \odot}+\Delta \alpha_{\mathrm{MLT}}$ and an isochrone with $\alpha_{\mathrm{MLT}, \odot}$. Positive values of $\Delta(B-V)$ correspond to $\Delta \alpha_{\mathrm{MLT}}=-0.2$, negative values to $\Delta \alpha_{\mathrm{MLT}}=+0.2$. The continuous line is for $[\mathrm{Fe} / \mathrm{H}]=0.0$, dot-dash line for $[\mathrm{Fe} / \mathrm{H}]=-1.0$ and dash line for $[\mathrm{Fe} / \mathrm{H}]=+0.14$ dex (Hyades).

\subsection{Lower part of the $M S$}

There is a poor fit of the observed data in the cooler regions of the HR diagram (i.e. for $(B-V) \gtrsim 1.2 \mathrm{mag}, T_{\text {eff }} \lesssim$ $4300 \mathrm{~K})$. The discrepancy in $(B-V)$ between models and observations amounts to about $0.07 \mathrm{mag}(0.10 \mathrm{mag})$ at $(B-V) \sim 1.3 \mathrm{mag}(1.4 \mathrm{mag})$.

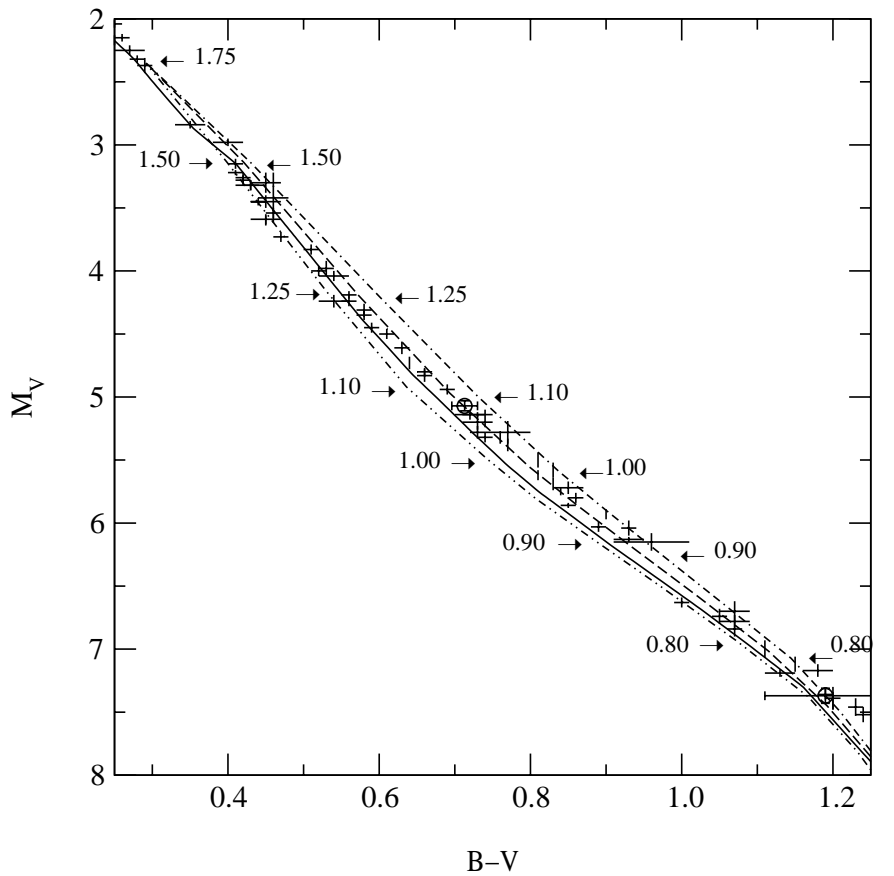

Fig. 10. Effect of a change of $\alpha_{\mathrm{MLT}}$ on the MS position at Hyades metallicity. Isochrones aged $625 \mathrm{Myr}$, $[\mathrm{Fe} / \mathrm{H}]=0.14, Y=0.26$, are plotted: the continous isochrone is for $\alpha_{\mathrm{MLT}, \odot}$, dashed for $\alpha_{\mathrm{MLT}}=\alpha_{\mathrm{MLT}, \odot}-0.2$, dotdashed for $\alpha_{\mathrm{MLT}}=\alpha_{\mathrm{MLT}}-0.4$, and dot-dot-dashed for $\alpha_{\mathrm{MLT}}=\alpha_{\mathrm{MLT}, \odot}+0.2$. The positions of models of different masses on the isochrones are indicated by arrows. Open circles are $\mathrm{vB} 22 \mathrm{~A}$ and $\mathrm{vB} 22 \mathrm{~B}$.

In the low-mass MS star region both the observations and the models presented here become less secure. Errors in the equation of state or external boundary conditions may have an important impact on the models. On the other hand, the $B-V$ color index is not the best index to be used in the low mass red dwarf region. However, we found the same kind of discrepancy in other open clusters both in $(B-V)$ and $(V-I)$ (Robichon et al. 1999; Lebreton 2000). We now examine the input physics of the models.

\subsubsection{Equation of state}

In most models, we used the CEFF EOS (see Sect. 4). Sophisticated EOSs, including collective effects, have been designed to study the Sun (MHD EOS, Mihalas et al. 1988; OPAL EOS, Rogers et al. 1996) and very-low mass stars and planets (as the SC EOS, Saumon \& Chabrier 1991). The effects of the EOS on the models are illustrated in Fig. 11 where we compare models calculated with the CEFF and OPAL EOSs. Below $0.65 M_{\odot}$, the MS slope is steeper in models including the most sophisticated EOSs and the fit with the observed low MS position becomes worse. We also calculated models with the MHD and SC EOS which exhibit the same behaviour.

Lebreton \& Däppen (1988) have shown that the M$\mathrm{L}$ relationship is very sensitive to the EOS used at low 
mass. We calculated models with CEFF, MHD, OPAL and $\mathrm{SC}$ and found that at solar metallicity a model of $\sim 0.50 M_{\odot}$ is $\approx 0.75 \mathrm{mag}$ brighter when calculated with the MHD, OPAL or SC EOS instead of the CEFF EOS. In the Hyades, no binaries have yet been observed in this mass range, but in the future new constraints for the physics will certainly come from low-mass binaries.

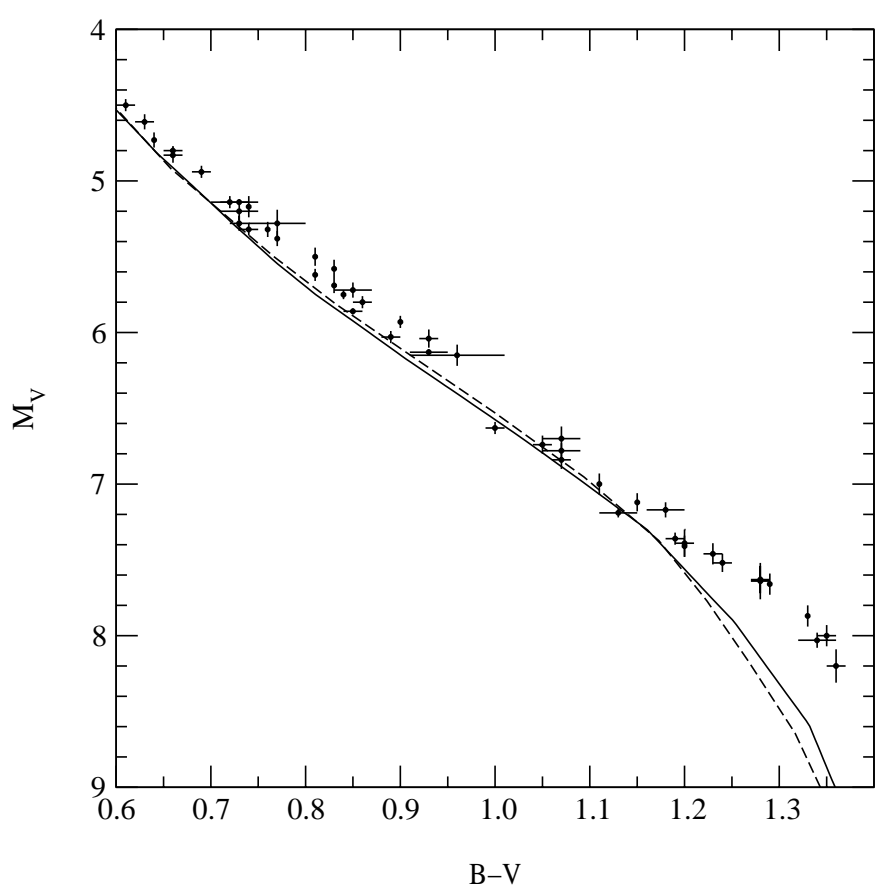

Fig. 11. Effect of the EOS on the lower MS position. The continuous line is the isochrone calculated with the CEFF EOS; dashed line with the OPAL EOS.

\subsubsection{Model atmospheres}

Model atmospheres are fundamental because they fix the external boundary conditions of the interior models and intervene in the transformations of the model results from the $\left(M_{\mathrm{bol}}, T_{\mathrm{eff}}\right)$-plane to the $\left(M_{V}, B-V\right)$ colourmagnitude diagram.

The BaSeL conversions we used to convert the $\left(M_{\mathrm{bol}}\right.$, $\left.T_{\text {eff }}\right)$ values to $\left(M_{V}, B-V\right)$ are based on Kurucz's ATLAS9 model atmospheres down to $T_{\text {eff }} \sim 3500 \mathrm{~K}$ and on Allard \& Hauschildt (1995) non-grey model atmospheres beyond. Kurucz's ATLAS9 models are known to be insufficient below $4500 \mathrm{~K}$ but from preliminary tests we do not expect that conversions based on the new model atmospheres of Hauschildt et al. (1999) will improve the fit in the 4000-4500 K range. Castellani et al. (2000) examined three sets of conversions (either empirical or based on Kurucz's models) and did not find improvement of the slope-fitting at high $(B-V)$. We also examined various empirical conversions and reach the same conclusions.

On the other hand, the boundary conditions of our models are derived from the $T(\tau)$-laws based on Eddington's grey model atmosphere. As demonstrated by
Chabrier \& Baraffe (1995), because convection penetrates into optically thin layers in the envelope of low-mass stars, it is not correct to use grey model atmospheres and related $\mathrm{T}(\tau)$-laws to fix the boundary conditions of the interior models for $T_{\text {eff }} \lesssim 4000 \mathrm{~K}$. Chabrier \& Baraffe find that, at solar metallicity and in the range $\sim 3000-4000 \mathrm{~K}$, models based on the Eddington $\mathrm{T}(\tau)$-laws are hotter and brighter than models based on non-grey full model atmospheres, the $T_{\text {eff-difference is of } \sim 50 \mathrm{~K} \text { at } T_{\text {eff }} \sim 3800 \mathrm{~K}}$ and reaches $\sim 200 \mathrm{~K}$ at $T_{\text {eff }} \sim 3300 \mathrm{~K}$ (their Fig. 2 ).

We have here a discrepancy in $(B-V)$ that amounts to $0.07-0.10 \mathrm{mag}$ at $B-V \sim 1.3 \mathrm{mag}(1.4 \mathrm{mag})$. From the BaSeL transformations we estimate that at $M_{V} \sim 8$., the effective temperature of our models (which is $\sim 4150 \mathrm{~K}$ ) should be reduced by more than $250 \mathrm{~K}$ to increase the $(B-V)$ index to the observed position. This appears to be very large with respect to Chabrier \& Baraffe's results.

At high temperatures $(\gtrsim 4500 \mathrm{~K})$, models calculated with outer boundary conditions taken from $T(\tau)$-laws based on Kurucz's model atmospheres and models using Eddington's grey law differ by less than $0.01 \mathrm{mag}$ in $(B-V)$.

\subsection{Turn-off region and age}

The turn-off region in the Hyades corresponds to $M_{V^{-}}$ values below 2.5 mag. As shown in Fig. 12 there are only 12 turn-off stars among the secure stars selected by de Bruijne et al. (2000). We have added to the figure the two components of $\theta^{2}$ Tau and the brighter component of the 51 Tau system.

The interpretation of observations in the turn-off region of the Hyades are complicated by the effects of rotation and overshooting that make either models or photometric data uncertain.

\subsubsection{Overshooting}

Hyades stars at turn-off have masses in the range 2.0$2.5 M_{\odot}$. Therefore, they have had convective cores during their MS phase. Overshooting of convective cores extends the mixing in central regions: it increases the amount of hydrogen available for H-burning and in turn, increases the MS lifetime and the MS width in the HR diagram. The amount of overshooting is poorly known. Recently, Ribas et al. (2000) proposed a mass dependence of the overshooting on the basis of the modeling of 8 eclipsing binaries. At $M \simeq 2 M_{\odot}$ and for solar metallicity, they give $d_{\mathrm{ov}}=0.17 \pm 0.05 H_{\mathrm{p}}$ which is fully compatible with $d_{\mathrm{ov}}=0.2 H_{\mathrm{p}}$, the value of Schaller et al. (1992) adopted here.

As discussed in Perryman et al. (1998) who compared two sets of isochrones with and without overshooting, it is possible to fit the turn-off in both cases at different ages. This is shown again in Fig. 12 where we have plotted isochrones of various ages with and without overshooting. The theoretical isochrones without overshooting are the 


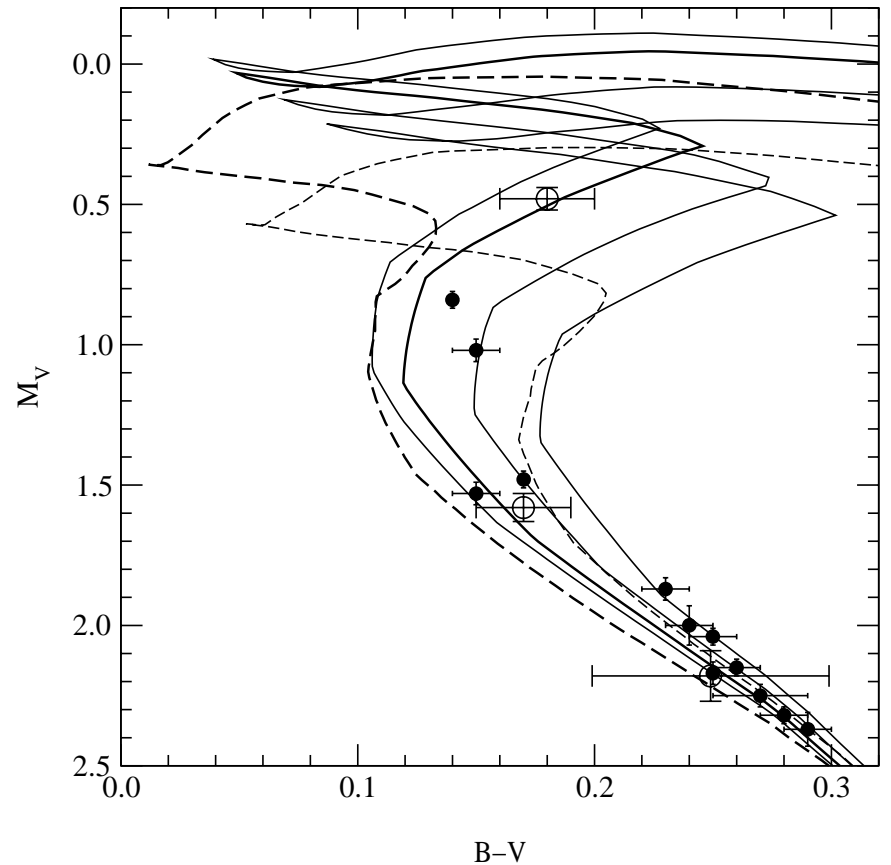

Fig. 12. Zoom on the turn-off region. The observational data are the same as in Fig 5. Isochrones with $([\mathrm{Fe} / \mathrm{H}]=0.14$, $Y=0.26)$ and different ages are plotted. From left to right: continous lines are isochrones with overshooting and aged $600 \mathrm{Myr}, 625 \mathrm{Myr}$ (bold), $700 \mathrm{Myr}$ and $750 \mathrm{Myr}$, dashed lines are isochrones without overshooting and aged $550 \mathrm{Myr}$ (bold) and 600 Myr.

same as in Perryman et al. (1998) but converted into the $\left(M_{V}, T_{\text {eff }}\right)$ plane by means of the BaSeL library. For the same $(B-V)$ at turn-off, models without overshooting are about $100 \mathrm{Myr}^{2}$ younger than models with $d_{\mathrm{ov}}=0.2 H_{\mathrm{p}}$. Models without overshooting appear to give a poorer fit of the position of $\theta^{2}$ Tau A, and at the magnitude of $\theta^{2}$ Tau A, they predict a mass 2 percent larger than the mass predicted by models with overshooting. However, the mass of $\theta^{2}$ Tau A is not known with enough precision to discriminate between models with $d_{\mathrm{ov}}=0.2 H_{\mathrm{p}}$ and models without overshooting. Furthermore, as will be discussed in Sect. 6.5.2, one must also account for rotation effects in the Hyades turn-off region.

\subsubsection{Rotation}

The turn-off region of the Hyades also corresponds to the $\delta$ Scuti instability strip. In that region, stars are mainly rapid rotators and variable stars and, some of them have been identified as $\delta$-Scuti pulsators (Antonello \& Pasinetti Fracassini 1998). The 15 stars seen in Fig. 12 have $v_{\mathrm{e}} \sin i$ in the range $43-175 \mathrm{kms}^{-1}$ (Bernacca \&

\footnotetext{
${ }^{2}$ Our models are based on $\sim 700$ mass shells. Breger \& Pamyatnykh (1998) pointed out that the MS width is reduced if models are calculated with smaller time and space steps, the effect being larger in models with overshooting. This numerical effect could result in an overestimate of the ages in the turn-off region of $\sim 50 \mathrm{Myr}$.
}

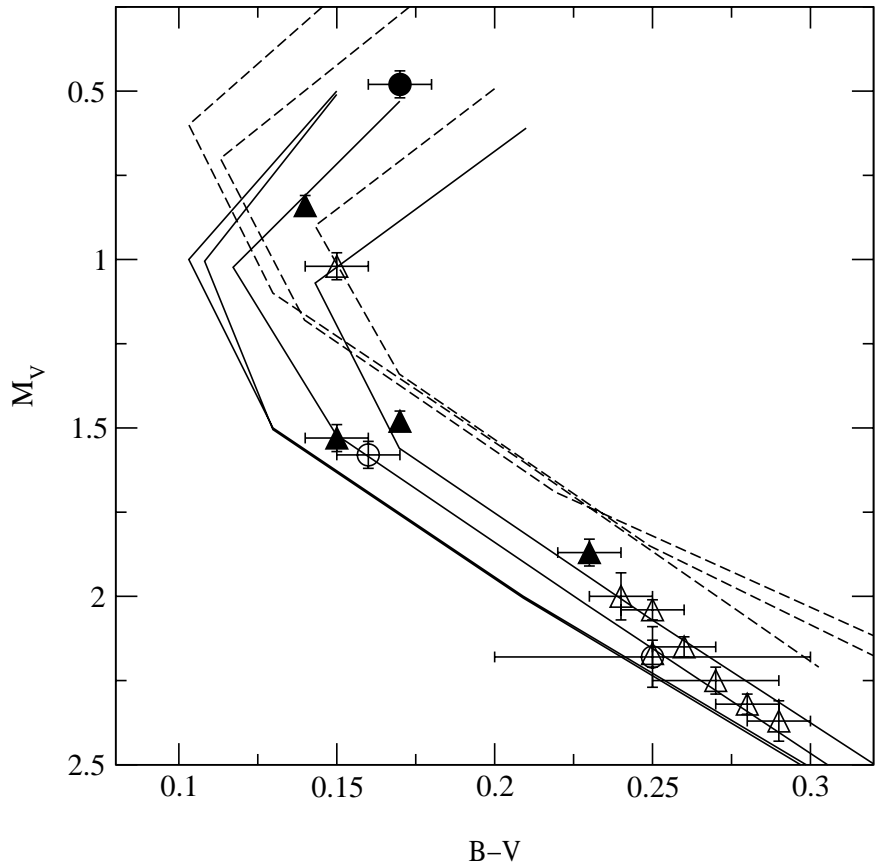

Fig. 13. Effects of rotation on photometric data. Continuous lines are isochrones with from left to right $v_{\mathrm{e}} \sin i=$ $0,50,100,150 \mathrm{kms}^{-1}$ and $i=90$ degrees. Dashed lines are ischrones with, from left to right (at $M_{V}=0.5$ ), $v_{\mathrm{e}} \sin i=$ $50,100,150 \mathrm{~km} \mathrm{~s}^{-1}$ and $\omega=0.95$ (see text). Open (full) symbols are Hyades with $v_{\mathrm{e}} \sin i>90 \mathrm{~km} \mathrm{~s}^{-1}\left(v_{\mathrm{e}} \sin i<90 \mathrm{~km} \mathrm{~s}^{-1}\right)$. Circles are for the binaries studied in Sect. 3.

Perinotto 1970; Abt \& Morrell 1995) and among these stars, 10 stars have $v_{\mathrm{e}} \sin i \gtrsim 90 \mathrm{~km} \mathrm{~s}^{-1}$ ( $v_{\mathrm{e}}$ is the equatorial velocity and $i$ the inclination of the rotation axis with respect to the line of sight). In particular, 51 Tau A has measured values of $v_{\mathrm{e}} \sin i$ in the range $97-125 \mathrm{~km} \mathrm{~s}^{-1}$, $\theta^{2}$ Tau A (which is a well-known $\delta$-Scuti) has $v_{\mathrm{e}} \sin i \sim$ $80 \mathrm{~km} \mathrm{~s}^{-1}$ while its companion $\theta^{2}$ Tau B rotates even faster with published $v_{\mathrm{e}} \sin i$ in the range $90-170 \mathrm{~km} \mathrm{~s}^{-1}$ (Torres et al. 1997c).

Rotation complicates the interpretation of the HR diagram and makes the age estimates uncertain. It affects both the models and the photometric data.

- It has been known for a long time that rotation produces a displacement of the stars in any photometric $\mathrm{HR}$ diagram by amounts depending on $v_{\mathrm{e}}$ and $i$ (Maeder \& Peytremann 1972). Pérez-Hernández et al. (1999) have computed the photometric magnitudes of rotating atmospheres. They give the magnitude differences between non-rotating stars of given $\left(T_{\text {eff }}, \log g\right)$ and their rotating copartners, as a function of the $i$ angle and of $\omega=\Omega / \Omega_{\mathrm{c}}$ where $\Omega$ is the star angular velocity and $\Omega_{\mathrm{c}}=\left(8 G M / 27 R_{\mathrm{p}}^{3}\right)^{1 / 2}(M$ is the mass, $R_{\mathrm{p}}$ is the polar radius). Interpolation in their Table 2 at $\left(T_{\text {eff }}, \log g\right)$-values of the model isochrones yields the corrections $\Delta M_{V}$ and $\Delta(B-V)$ to apply to our $M_{V}$ and $(B-V)$-values. We calculated the corrections for three values of $v_{\mathrm{e}} \sin i\left(50,100\right.$ and $\left.150 \mathrm{~km} \mathrm{~s}^{-1}\right)$, and for each $v_{\mathrm{e}} \sin i$, we considered the case where 
$i=90$ degrees ( $v_{\mathrm{e}}$ and therefore $\omega$ are minimum), and the case where $\omega=0.95$ ( $v_{\mathrm{e}}$ tends to the maximum allowed value, $i$ tends to its minimum possible value). The results are plotted in Fig. 13. When the rotation rate is close to maximum, the isochrone is shifted both toward higher luminosities and smaller effective temperatures. The shift in $(B-V)$ is of a few tenths of a magnitude and does not depend much on $v_{\mathrm{e}} \sin i$, except in the upper part of the turn-off. For minimum rotation rates ( $i=90$ degrees), the effect of rotation is to shift the isochrones, mainly in $(B-V)$, by an amount that increases with $v_{\mathrm{e}}$. Rotating stars are therefore expected to be spread in a band that extends on the red side of the non-rotating isochrone. A detailed individual analysis of each star at turn-off is beyond the scope of this work. However, with the help of Fig. 12, we can estimate that the effect of rotation on photometry could be responsible for an overestimate of the age of the Hyades by $\sim 50 \mathrm{Myr}$.

- On the other hand, models are modified when rotation is taken into account. Breger \& Pamyatnykh (1998) showed that models with uniform rotation are displaced toward the right in the HR diagram, in the same way as models including overshooting. Furthermore, the rotation profile inside a star is related to the redistribution of the angular momentum by internal motions which could be generated by meridional circulation and shear turbulence in a rotating medium (see Zahn 1992). Such motions might induce internal mixing. As shown by Talon et al. (1997), in the HR diagram these rotational effects also "mimic" overshooting (for instance in a $9 M_{\odot}$ star with a rotational velocity of $100 \mathrm{~km} \mathrm{~s}^{-1}$ the effect is roughly equivalent to the effect of an overshooting of $0.2 H_{\mathrm{p}}$ ).

Overshooting and rotation have similar effects on model isochrones and therefore similar signatures in the HR diagram. These effects cannot be discriminated with the present data; additional data on the internal structure of the considered stars are required that will hopefully (and only) be brought by asteroseismology measurements. On the basis of the present data and model isochrones, the upper limit on the Hyades age is around $\sim 650 \mathrm{Myr}$, the lower limit would be $\sim 500$ Myr (no overshooting, rotation corrections on photometry included).

\section{Summary and conclusions}

High accuracy observations including astrometric, photometric and spectroscopic data have been gathered for the nearest open cluster, the Hyades. These data provide precious information: the Hyades is the only cluster in which individual distances are available for a bunch of a hundred stars and in which individual masses have been measured in several binary systems. These strong constraints have been used to infer the characteristics of the cluster from the comparison with stellar models.
The helium abundance of the cluster can be constrained by the M-L relation provided (1) the stellar data are accurate enough, (2) the error on the bolometric corrections needed to convert the model results in the observed M-L plane are small and, (3) the input physics of the models is well chosen. The only Hyades binary system with data accurate enough to constrain the helium abundance is vB22. We estimated the vB22 helium abundance using models including different input physics (EOS, atmospheres, diffusion, PMS) and we allowed variations of $\alpha_{\mathrm{MLT}}$ of \pm 0.4 around the solar value. The result, $Y=0.255 \pm 0.009$, implies that the Hyades have less helium than expected from their supersolar metallicity. A low helium abundance has already been suggested in the past (see for instance VandenBerg \& Poll 1989; Torres et al. 1997c) and, a long time ago, on the basis of photometric measurements, Hyades stars were found to be helium deficient with respect to field stars of same metallicity (Strömgren et al. 1982).

We pointed out that the error bar on $Y$ is much smaller if $[\mathrm{Fe} / \mathrm{H}]$ is taken from photometry (internal error bar on $[\mathrm{Fe} / \mathrm{H}]$ around 10 percent, Grenon 2000) rather than from spectroscopy (internal error bar on $[\mathrm{Fe} / \mathrm{H}]$ around 35 percent, Cayrel et al. 1997). Progress will come from a better description of the so-called metallicity by the future determinations of the individual abundances of the main metallicity contributors. The $\mathrm{C}, \mathrm{N}, \mathrm{O}$ abundances are presently poorly known while the Ne abundance is unknown (R. Cayrel, private communication). On the other hand, it would be worthwhile to improve the mass values in the four other binary systems which are presently not good enough to constrain models (and helium) significantly.

An upper limit of $\sim 650 \mathrm{Myr}$ on the cluster age can be estimated from the positions of stars at turn-off. It is difficult to estimate precisely the age of the Hyades because at turn-off rapidly rotating stars are found which had a convective core on the MS. Rotation affects the photometry and makes the observed HR diagram positions uncertain, whereas internal rotation and overshooting are responsible for internal mixing that may change the model course in the HR diagram. As a result, the age of the Hyades might be in the range 500-650 Myr. An improvement of the mass of $\theta^{2}$ Tau A would certainly better constrain the overshooting by anchoring the star more precisely on its isochrone. Asteroseismological measurements and analysis are required to get a better understanding of the transport processes inside stars (Goupil et al. 2000).

The observed MS slope, in the region of the HR diagram corresponding to masses in the range $0.8-1.5 M_{\odot}$, is quite well-defined and provides first tests of the $\alpha_{\mathrm{MLT}^{-}}$ value to be used in models. It suggests that $\alpha_{\mathrm{MLT}}$ could be a slowly decreasing function of mass: $\alpha_{\mathrm{MLT}}$ would decrease from values of $\sim 1.8-1.9$ at high mass to values of $\sim 1.2-1.4$ at low mass. The confirmation of that trend would require extremely precise radii or $T_{\text {eff }}$ for individual stars along the MS. Presently, both radii and $T_{\text {eff }}$ are only available for vB22 (only vB22A sits in the HR diagram region sensitive 
to $\left.\alpha_{\mathrm{MLT}}\right)$ and since they do not agree, we have not been able to draw firm constraints on $\alpha_{\mathrm{MLT}}$ from these data.

In the very low mass region of the HR diagram, models are definitely too blue. This region provides tests on the model external boundary conditions and on the equation of state and the knowledge of a few masses would help to understand the origin of the discrepancy.

The Hyades stars are now better understood because they are fully seen as individuals and because of the considerable recent progress in the description of the physics of the stellar plasma (opacities, EOS). In 1989, VandenBerg \& Poll (1989) used stellar models to infer the Hyades distance modulus by means of the MS fitting technique assuming that the helium content was solar. Now that we have removed the distance problem, we are able to test finer details in the models. We can hope that in the decade to come, a substantial number of open clusters will come to be known at the level of accuracy presently reached for the Hyades and even better. Hipparcos provided mean distances to a few open clusters which already give rise to many questions. However, the discussion of the helium abundance in the nearer clusters is limited because errors sources can be important (color-calibrations and bolometric corrections, distances, metallicity) and because of the lack of well observed binaries (Robichon et al. 1999; Lebreton 2000). In the future, GAIA will determine individual distances of stars in many clusters together with information on their abundances (Perryman et al. 1997): it is expected that about 120 clusters, located to within $1 \mathrm{kpc}$, will reach to the level of accuracy or even better than what is reached today in the Hyades. GAIA will also discover binary systems in which it will measure masses accurately (at least ten binary systems are expected per cluster). Other observational devices will also provide accurate masses and radii in the near future. Therefore, we can expect that we shall go on constraining the models to progress in the understanding of the chemical and dynamical evolution of our Galaxy.

Acknowledgements. We warmly thank D. Dravins, L. Lindegren and J. de Bruijne who provided their parallax data. We are grateful to A. Baglin, G. Cayrel de Strobel, R. Cayrel, H.-G. Ludwig, F. Arenou, M. J. Goupil and E. Michel for many fruitful discussions. We thank the referee Dr. A. A. Pamyatnykh for interesting remarks and information. Y. Lebreton acknowledges University of Rennes 1 for working facilities. This work was partially supported by the "Convénio ICCTI Embaixada de França, number 060/B0" and by the project "ESO/P/PRO/12128/1999" from "Fundaço para a Ciência e Tecnologia".

\section{References}

Abt, A. H., \& Morrell, N. I. 1995, ApJS, 99, 135

Alexander, D. R., \& Ferguson, J. W. 1994, ApJ, 437, 879

Allard, F., \& Hauschildt 1995, ApJ, 445, 433

Hauschildt, P. H., Allard, F., \& Baron, E. 1999, ApJ, 512, 377

Alonso, A., Arribas, S., \& Martínez-Roger, C. 1995, A\&A, 297, 197
Anders, E., \& Grevesse, N. 1989, Geochim. Cosmochim. Acta, 53, 197

Andersen, J. 1991, AAR, 3, 91

Antonello, E., \& Pasinetti Fracassini, L. E. 1998, A\&A, 331, 995

Bernacca, P. L., \& Perinotto, M. 1970, A catalogue of stellar rotational velocities, Contr. Oss. Astrof. Padova in Asiago, 239

Bessell, M. S., Castelli, F., \& Plez, B. 1998, A\&A, 333, 231

Böhm-Vitense, E. 1958, Z. Astrophys., 54, 114

Breger, M., \& Pamyatnykh, A. A. 1998, A\&A, 332, 958

de Bruijne, J. H. J., Hoogerwerf, R., \& de Zeeuw, P. T. 2001, A\&A, 367, 111

Castellani, V., degl'Innocenti, S., \& Prada Moroni, P. G. 2001, MNRAS, 320, 66

Caughlan, G. R., \& Fowler, W. A. 1988, Atomic Data Nuc. Data Tables, 40, 284

Cayrel, R., Castelli, F., Katz, D., et al. 1997, in Hipparcos Venice '97, ESA SP-402, 433

Cayrel de Strobel, G., Crifo, F., \& Lebreton, Y. 1997, in Hipparcos Venice '97, ESA SP-402, 687

Cayrel de Strobel, G., Cayrel, R., Lebreton, Y., \& Morel, P. 2001, A\&A, in preparation

Chabrier, G., \& Baraffe, I. 1995, ApJ, 451, L29

Christensen-Dalsgaard, J. 1991, in Challenges to theories of the structure of moderate-mass stars, ed. D. O. Gough, \& J. Toomre (Springer-Verlag), 11

Dravins, D., Lindegren, L., Madsen, S., \& Holmberg, J. 1997, in Hipparcos Venice '97, ESA SP-402, 733

Eggleton, P. P., Faulkner, J., \& Flannery, B. P. 1973, A\&A, 23, 325

Fernandes, J., Lebreton, Y., Baglin, A., et al. 1998, A\&AS, 338,455

Goupil, M.-J., Dziembowski, W. A., Pamyatnykh, A. A., \& Talon, S. 2000, in Delta Scuti and Related Stars, ed. M. Breger, \& M. Montgomery, ASP Conf. Ser., 210, 267

Grevesse, N., \& Noels, A. 1993, in Origin and Evolution of the Elements, ed. N. Prantzos, E. Vangioni-Flam, \& M. Cassé (Cambridge: Cambridge University Press)

Grenon, M. 2000, in Luminosity Calibrations with Hipparcos, Highlights of Astronomy Vol. 12, Joint Discussion 13 of the XXIIIIrd General Assembly of the IAU, ed. T. L. Evans, in press

Iglesias, C. A., \& Rogers, F. J. 1996, ApJ, 464, 943

Kurucz, R. L. 1991, in Stellar Atmospheres: Beyond Classical Models, ed. L. Crivellari, I. Hubeny, \& D. G. Hummer, Nato ASI Ser. (Dordrecht: Kluwer), 441

Lastennet, E., Valls-Gabaud, D., Lejeune, Th., \& Oblak, E. 1999, A\&A, 349, 485

Lebreton, Y. 2000, in Hipparcos and the Luminosity Calibration of the Nearer Stars, 24th IAU GA, JD 13, Highlights of Astronomy, vol. 12, ed. T.L. Evans (Kluwer)

Lebreton, Y., \& Däppen, W. 1988, in Proc. Symp. Seismology of the Sun and Sun-like Stars, Tenerife, Spain, ESA SP-286, 661

Lebreton, Y., Gómez, A. E., Mermilliod, J.-C., \& Perryman, M. A. C. 1997, in Hipparcos Venice '97, ESA SP-402, 231

Lebreton, Y., Perrin, M.-N., Cayrel, R., Baglin, A., \& Fernandes, J. 1999, A\&A, 350, 587

Lejeune, T. 2001, in preparation

Lejeune, T., \& Buser, R. 1999, in Spectrophotometric dating of stars and galaxies, ed. I. Hubeny, S. Heap, \& R. Cornett, ASP Conf. Ser., 192, 211 
Lejeune, T., Cuisinier, F., \& Buser, R. 1997, A\&AS, 125, 246 Lejeune, T., Cuisinier, F., \& Buser, R. 1998, A\&AS, 130, 75 Ludwig, H. G., Freytag, B., \& Steffen, M. 1999, A\&A, 346,111 Meader, A., \& Peytremann, E. 1972, A\&A, 21, 279

Martin, C., Mignard, F., Hartkopf, W. I., \& McAlister, H. A. 1998, A\&AS, 133, 149

McClure, R. D. 1982, ApJ, 254, 606

Mermilliod, J.-C. 1998, in Dynamical Studies of Star Clusters and Galaxies, Joint European and National Astronomy Meeting for 1998 (JENAM98), ed. P. Kroupa, J. Palous, \& R. Spurzem, Abstract Book published by ESA, The Netherlands

Mihalas, D., Däppen, W., \& Hummer, D. G. 1988, ApJ, 331, 815

Morel, P. J. 1997, A\&AS, 124, 597

Narayanan, V. K., \& Gould, A. 1999, ApJ, 515, 256

Nissen, P. E. 1976, A\&A, 50, 343

Peimbert, M., Peimbert, A., \& Ruiz, M. T. 2000, ApJ, 541, 688

Pérez-Hernández, F., Claret, A., Hernández, M. M., \& Michel, E. 1999, A\&A, 346, 586

Perryman, M., Brown, A. G. A., Lebreton, Y., et al. 1998, A\&A, 331, 81

Perryman, M. A. C., Lindegren, L., \& Turon, C. 1997, in Hipparcos Venice '97, ESA SP-402, 743

Peterson, D. M., \& Solensky, R. 1988, ApJ, 333, 256

Peterson, D. M., Stefanik, R. P., \& Latham, D. W. 1993, AJ, 105,2260
Proffitt, C. R., \& VandenBerg, D. A. 1991, ApJS, 77, 473

Ribas, I., Jordi, C., \& Giménez, A. 2000, MNRAS, 318, L55

Robichon, N., Arenou, F., Lebreton, Y., Turon, C., \& Mermilliod, J.-C. 1999, in Harmonizing Cosmic Distance Scales in a Post-HIPPARCOS Era, ed. D. Egret, \& A. Heck, ASP Conf. Ser., 167, 72

Rogers, F. J., Swenson, F. J., \& Iglesias, C. A. 1996, ApJ, 456, 902

Saumon, D., \& Chabrier, G. 1991, Phys. Rev. A, 44, 5122

Schaller, G., Schaerer, D., Meynet, G., \& Maeder, A. 1992, A\&AS, 96, 269

Schiller, S. J., \& Milone, E. F. 1987, AJ, 93, 1471

Schwan, H. 1991, A\&A, 243, 386

Söderhjelm, S. 1999, A\&A, 341, 121

Strömgren, B., Olsen, E. H., \& Gustafsson, B. 1982, PASP, 94,5

Talon, S., Zahn, J.-P., Maeder, A., \& Meynet, G. 1997, A\&A, 322, 209

Torres, G., Stefanik, R. P., \& Latham, D. W. 1997a, ApJ, 474, 256

Torres, G., Stefanik, R. P., \& Latham, D. W. 1997b, ApJ, 479, 268

Torres, G., Stefanik, R. P., \& Latham, D. W. 1997c, ApJ, 485, 167

VandenBerg, D. A., \& Poll, H. E. 1989, AJ, 98, 1451

Zahn, J. P. 1992, A\&A, 265, 115 